\title{
Combed 3-Manifolds with Concave Boundary, Framed Links, and Pseudo-Legendrian Links
}

\author{
Riccardo Benedetti Carlo Petronio
}

November 8, 2018

\begin{abstract}
We provide combinatorial realizations, according to the usual objects/moves scheme, of the following three topological categories: (1) pairs $(M, v)$ where $M$ is a 3-manifold (up to diffeomorphism) and $v$ is a (non-singular vector) field, up to homotopy; here possibly $\partial M \neq \emptyset$, and $v$ may be tangent to $\partial M$, but only in a concave fashion, and homotopy should preserve tangency type; (2) framed links $L$ in $M$, up to framed isotopy; (3) triples $(M, v, L)$, with $(M, v)$ as above and $L$ transversal to $v$, up to pseudo-Legendrian isotopy (transversalitypreserving simultaneous homotopy of $v$ and isotopy of $L$ ). All realizations are based on the notion of branched standard spine, and build on results previously obtained. Links are encoded by means of diagrams on branched spines, where the diagram is $\mathrm{C}^{1}$ with respect to the branching. Several motivations for being interested in combinatorial realizations of the topological categories considered in this paper are given in the introduction. The encoding of links is suitable for the comparison of the framed and the pseudo-Legendrian categories, and some applications are given in connection with contact structures, torsion and finite-order invariants. An estension of Trace's notion of winding number of a knot diagram is introduced and discussed.
\end{abstract}

Mathematics Subject Classification (1991): 57N10 (primary), 57M25, 57R25 (secondary).

\section{Introduction}

This paper describes combinatorial realizations, based on the machinery of branched standard spines (see Section 1) of the following three topological categories (in which manifolds and diffeomorphisms are oriented by default):

1. Combed 3-manifolds with concave boundary, that is pairs $(M, v)$, where $M$ is a compact 3-manifold (possibly with boundary), and $v$ is a nowhere-zero vector field on $M$ with simple tangency circles of concave type on $\partial M$, up to diffeomorphism of $M$ and homotopy of $v$ through fields of the same sort; 
2. Framed links in 3-manifolds, that is pairs $(M, L)$, where $M$ is as above and $L$ is a framed link in $M$, up to diffeomorphism of $M$ and framed isotopy of $L$;

3. Pseudo-Legendrian links in combed 3-manifolds, that is triples $(M, v, L)$, where $(M, v)$ is as above and $L$ is transversal to $v$, up to diffeomorphism of $M$ and 'pseudo-Legendrian isotopy' of $(v, L)$, i.e. simultaneous homotopy of $v$ and isotopy of $L$ through pairs $(v, L)$ of the same type.

We will denote these categories respectively by Comb, Fram and PLeg. (Regarding names, recall that a non-zero vector field up to homotopy is often called a combing, and that if $\xi$ is an oriented contact structure and $L$ is Legendrian in $\xi$, then $\left(M, \xi^{\perp}, L\right)$ defines an element of PLeg.) Our realizations are given according to the by now popular scheme in 3-dimensional topology, namely:

(I) A class of combinatorial objects, each of which can be specified by a finite set of data, and a surjective reconstruction map which assigns to a combinatorial object a topological one;

(II) A finite set of local combinatorial moves on objects, finite combinations of which give the equivalence relation induced by the reconstruction map.

In the definitions of the topological categories given above we have been forced to include the action of diffeomorphisms, because we use spines, which determine manifolds only up to diffeomorphism. However if a certain manifold $M$ is given we can restrict to spines embedded in $M$ (rather than abstract ones), and get formally identical combinatorial realizations of the refined categories where only diffeomorphisms of $M$ isotopic to the identity are considered. We will mention how to do this in Section 2 for combings, but a similar refinement could easily be stated for framed links and for pseudo-Legendrian links.

Rather than providing precise statements of our realizations, in this introduction we give some general background and motivations, starting with Comb. A combinatorial realization of the subcategory $\mathrm{Comb}^{\mathrm{cl}}$ of Comb given by pairs $(M, v)$ with closed $M$ was given in [2]. In Section [2] we extend the arguments of [2] to the bounded case, and we actually refine the results proved there, by showing that some of the moves previously considered may actually be neglected. The realization of $\mathrm{Comb}^{\mathrm{cl}}$ in [2] was the basis for the treatment of other refinements of the category of 3-manifolds, involving spin structures and framings. These realizations proved fruitful in connection with spin-refined Turaev-Viro invariants (see Section 8.3 in [2]) and G. Kuperberg's invariants for combed and framed manifolds, of which a very constructive description is given in [5]. Our main motivation here comes from [4], where we have developed a theory of Euler structures with simple boundary and their Reidemeister-Turaev torsion (see [17], [18], [19]). The surjectivity of the reconstruction map of the realization of 
Comb was used in [4] to construct an explicit canonical $H_{1}$-equivariant bijection from the space of smooth Euler structures to the space of combinatorial Euler structures, and to exhibit a canonical Euler chain for the structure carried by a branched spine.

The subcategory of Fram consisting of framed links in closed manifolds was combinatorially realized by Turaev [16] in terms of link diagrams on a given standard spine, and moves on these diagrams (including the classical framed Reidemeister moves). In Section 3 we modify the situation considered by Turaev by taking a branched standard spine of the manifold, and restricting to link diagrams which are $\mathrm{C}^{1}$ with respect to the branching. On one hand, this allows to simplify the encoding of the framing, because the field carried by the spine is automatically transverse to the link, while Turaev needs to add half-twists. On the other hand, some technical complications emerge, because only $\mathrm{C}^{1}$ moves can be used. Nevertheless, a result formally analogous to Turaev's turns out to be true, yielding the presentation of Fram discussed in Section 3 . In Section 1 we exploit the fact that if a branched spine defines a global field on a manifold, according to the scheme given for Comb, then the link defined by a $\mathrm{C}^{1}$ diagram on the spine is automatically pseudo-Legendrian with respect to the field. This leads us to the presentation of PLeg.

Comparing the presentations of Fram and PLeg one notices a rather remarkable feature: the former is obtained from the latter just by adding the 'curl' (first Reidemeister) move. This fact has two interesting interpretations:

(a) it is a perfect combinatorial analogue of the imitation of a framed isotopy by a Legendrian isotopy in a contact manifold;

(b) it allows a partial extension of the notion of winding number of a link diagram.

The imitation mentioned in (a) plays a central role in the comparison, due to FuchsTabachnikov [8] and Tchernov [14] of framed and Legendrian finite-order invariants, and we believe that our combinatorial realizations could be of some help in the understanding of these invariants. In particular, we conjecture that the right environment in which finite-order invariants should be considered in precisely our category PLeg (we will provide an exact statement and some evidence in Section 5).

Concerning (b), recall first [15] that if a knot $K: S^{1} \rightarrow \mathbb{R}^{3}$ is transverse to the constant vertical field $\partial / \partial z$, then its equivalence class up to isotopy transverse to $\partial / \partial z$ is determined by the framed isotopy class and by the 'winding' number (the degree of $\pi \circ K^{\prime}$, where $\pi$ is the obvious projection on the horizontal unit circle). Using our presentations of Fram and Comb we can show that a partial analogue of this fact is true in any combed manifold with concave boundary, provided one allows a homotopy of the field simultaneous with the isotopy of the knot. In the general setting, however, the winding number only exists as a relative object, and we can prove that it leads to a well-defined invariant only under the assumption that the knot is 'good.' The notion of 'goodness' for knots emerged in our study of torsion as a relative invariant 
of pairs of pseudo-Legendrian knots which are framed-isotopic [4]. Many knots are good: for instance, all knots are good if the ambient manifold is a homology sphere, and most knots with hyperbolic complement are good. In Section 5 we will give some applications of the notion of relative winding number, in connection with torsion and finite-order invariants. In particular we will show the following:

Proposition 0.1. Let $M$ be a homology sphere, let $v$ be a field on $M$ and consider two pseudo-Legendrian knots in $(M, v)$ which are isotopic as framed knots. Then the following conditions are pairwise equivalent:

1. the knots are pseudo-Legendrian isotopic;

2. the relative winding number vanishes;

3. the knots have the same Maslov index;

4. the knots cannot be distinguished by the relative torsion invariants of [Q]

5. the knots are homotopic as pseudo-Legendrian immersions.

Moreover we will prove that torsion invariants cannot distinguish the pairs of framedisotopic Legendrian knots given in [14], which Tchernov shows to be distinguished by finite-order invariants.

We conclude by giving another perspective of the realization of PLeg. Recall that PLeg comes as a refinement of Turaev's presentation of Fram, which was the starting point of his beautiful theory of 4-dimensional shadows. We believe that the extra structure given by the branching of the spine, which underlies the presentation of PLeg, should have 4-dimensional counterparts. Our intuition is that "4-dimensional branched shadows", which are not quite defined yet, should correspond to $\operatorname{Spin}^{\mathrm{c}}$ structures on 4-manifolds and allow to treat their invariants. This intuition is supported by the fact that in dimension three branched spines indeed are a good framework to treat torsion of Euler structures (and hence, in particular, Seiberg-Witten invariants of closed 3manifolds with $\mathrm{Spin}^{\mathrm{c}}$ structures, see [18], [19], [12]).

Acknowledgment. Section 5 owes a lot to very useful discussions we had with Vladimir Tchernov.

\section{Branched spines and combings}

This section contains many definitions used below and reviews the theory developed in [Q] 



Figure 1: Convex (left) and concave (right) tangency to the boundary.

Manifolds and fields All the manifolds we will consider are 3-dimensional, oriented, and compact, with or without boundary. Using the Hauptvermutung, we will somewhat intermingle the differentiable and piecewise linear viewpoints. Maps will always respect orientations. All vector fields mentioned in this paper will be non-singular, and they will be termed just fields for the sake of brevity. A field $v$ on a manifold $M$ is called traversing if its orbits eventually intersect $\partial M$ transversely in both directions (in other words, orbits are compact intervals or points). A point where $v$ is tangent to $\partial M$ is called simple if it appears in a cross-section as in Fig. 1. The field is called concave if it is tangent to $\partial M$ only in a concave fashion, as shown on the left in the figure. Given a concave field $v$ on $M$, the boundary of $M$ naturally splits into the region on which $v$ points outside $M$ (which we denote by $B$ and call the black region), and the region on which $v$ points inside (denoted by $W$ and called white). Note that $\partial B=\partial W$ is a union of circles. The pair $(B, W)$, which is actually determined by any two of its elements, is called a boundary pattern on $M$. (This definition is a simplified version of that given in [4], because here we do not allow convex tangency.) Starting from the Poincaré-Hopf theorem one can show that a given boundary pattern $\mathcal{P}=(B, W)$ on $M$, i.e. a splitting of $\partial M$ into two surfaces with common boundary, arises from a concave field if and only if $\chi(W)=\chi(M)$. See [四].

Standard spines A simple polyhedron $P$ is a finite, connected, purely 2-dimensional polyhedron with singularity of stable nature (triple lines and points where six nonsingular components meet). Such a $P$ is called standard if all the components of the natural stratification given by singularity are open cells. Depending on dimension, we will call the components vertices, edges and regions.

A standard spine of a 3-manifold $M$ with $\partial M \neq \emptyset$ is a standard polyhedron $P$ embedded in $\operatorname{Int}(M)$ so that $M$ collapses onto $P$. Standard spines of oriented 3manifolds are characterized among standard polyhedra by the property of carrying an orientation, defined (see Definition 2.1.1 in [2]) as a "screw-orientation" along the edges (as in the left-hand-side of Fig. 2), with the obvious compatibility at vertices (as in the centre of Fig. 2). It is the starting point of the theory of standard spines that every oriented 3-manifold $M$ with $\partial M \neq \emptyset$ has an oriented standard spine, and can be reconstructed (uniquely up to equivalence) from any of its oriented standard spines. See [6] for the non-oriented version of this result and [1] or Proposition 2.1.2 in [2] for the (slight) oriented refinement. We will denote by $M(P)$ the manifold defined by $P$. Note that $\partial M(P) \neq \emptyset$. To recover closed manifolds one considers spines $P$ 

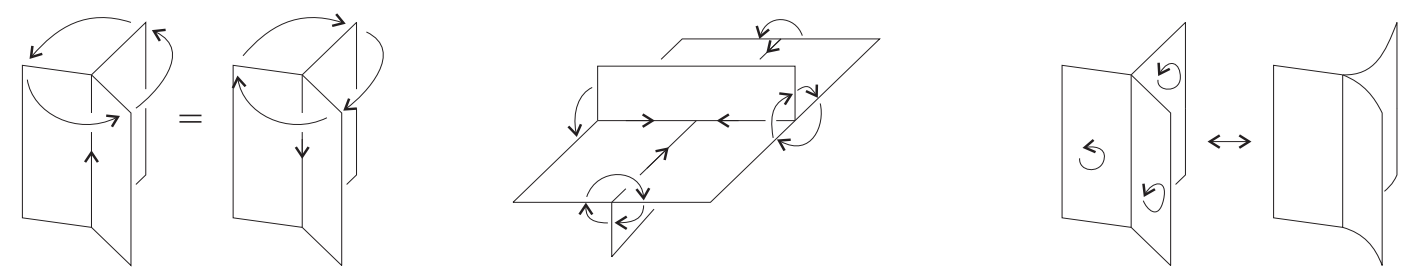

Figure 2: Convention on screw-orientations, compatibility at vertices, and geometric interpretation of branching.
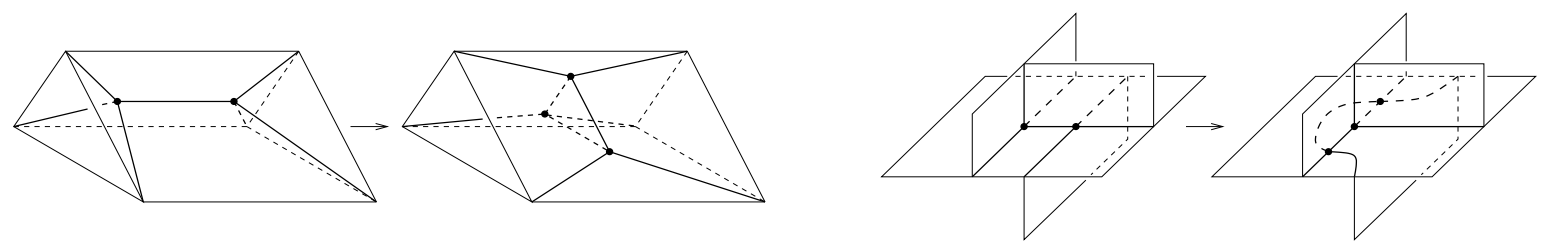

Figure 3: Two pictures of the Matveev-Piergallini move.

such that $\partial M(P) \cong S^{2}$, and defines $\widehat{M}(P)$ as $M(P) \sqcup_{f} D^{3}$ with $f: \partial M(P) \rightarrow S^{2}$ a diffeomorphism. Note that this definition makes sense also when $\partial M(P)$ has more than one component, but at least one is a sphere.

Moves for standard spines The fundamental move for standard spines, which (in both directions) preserves the topological type of the associated manifold, is the Matveev-Piergallini MP move, see [11], 13] and Fig. 3. Counting the vertices involved one is naturally led to call the positive MP a "2-to-3" move. The MP-move and its inverse are actually not sufficient to relate spines of the same manifold, because they obviously cannot apply to spines with one vertex. However, as soon as one decides to dismiss these "MP-rigid" spines (not the corresponding manifolds, which have plenty of other spines), the MP-move does become sufficient [13]. To deal with spines with one vertex the "0-to-2" move of Fig. $⿴$ (and its inverse) must be added.

Branched spines A branching on a standard polyhedron $P$ is an orientation for each region of $P$, such that no edge is induced the same orientation three times. See the right-hand side of Fig. 2 and Definition 3.1.1 in [2] for the geometric meaning of this notion. An oriented standard spine $P$ endowed with a branching is shortly named

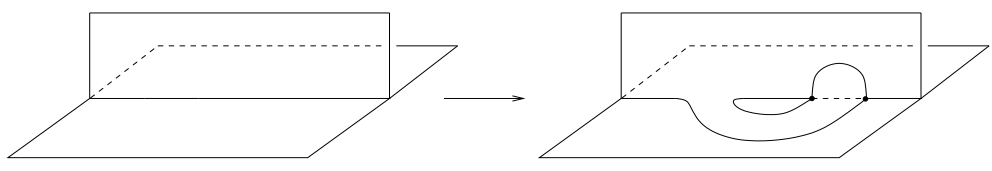

Figure 4: Another move on standard spines. 


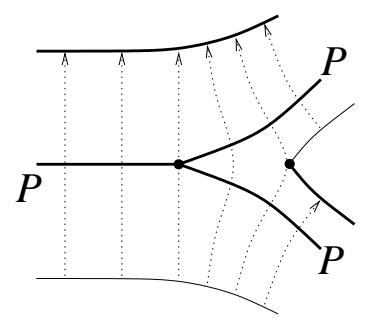

Figure 5: Manifold and field associated to a branched spine.

branched spine. We will never use specific notations for the extra structures: they will be considered to be part of $P$. The following result, proved as Theorem 4.1.9 in [2], is the starting point of our constructions.

Proposition 1.1. To every branched spine $P$ there corresponds a manifold $M(P)$ with non-empty boundary and a concave traversing field $v(P)$ on $M(P)$. The pair $(M(P), v(P))$ is well-defined up to equivalence, and an embedding $i: P \rightarrow \operatorname{Int}(M(P))$ is defined with the property that $v(P)$ is positively transversal to $i(P)$.

The topological construction which underlies this proposition is actually quite simple, and it is illustrated in Fig. 5. Concerning the last assertion of the proposition, note that the branching allows to define an oriented tangent plane at each point of $P$.

Non-traversing fields and closed manifolds As noted above, standard spines do not directly represent closed manifolds, but one can use spines of manifolds bounded by $S^{2}$ and cap off this sphere to get a closed manifold, or, viewing things the other way around, one can remove an open ball from a given closed manifold to get a bounded one. When one is interested in a manifold equipped with a field, one can try to use branched spines, but of course one sees that they are inadequate to give a direct description both when the manifold is closed and when the field is non-traversing. This limitation is circumvented again by removing a ball, with a proviso on the field on that ball.

Let $P$ be a branched standard spine, and assume that in $\partial M(P)$ there is only one component which is diffeomorphic to $S^{2}$ and is split by the tangency line of $v(P)$ to $\partial M(P)$ into two discs. Such a component will be denoted by $S_{\text {triv }}^{2}$. Now, notice that $S_{\text {triv }}^{2}$ is also the boundary of the closed 3-ball with constant vertical field, denoted by $B_{\text {triv }}^{3}$. This shows that we can cap off $S_{\text {triv }}^{2}$ by attaching a copy of $B_{\text {triv }}^{3}$, getting a compact manifold $\widehat{M}(P)$ and a concave field $\widehat{v}(P)$ on $\widehat{M}(P)$. If we denote by $\widehat{\mathcal{P}}(P)$ the boundary pattern of $\widehat{v}(P)$ on $\widehat{M}(P)$, we easily see that the pair $(\widehat{M}(P), \widehat{v}(P))$ is only well-defined up to diffeomorphism of $\widehat{M}(P)$ and homotopy of $\widehat{v}(P)$ through fields compatible with $\widehat{\mathcal{P}}(P)$. 

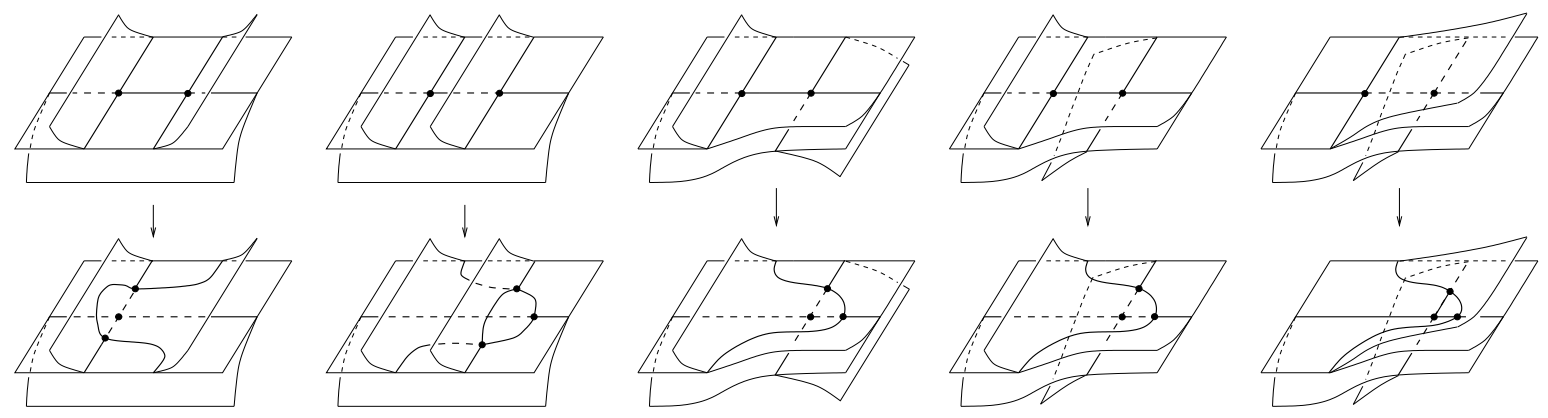

Figure 6: Sliding-MP-moves.

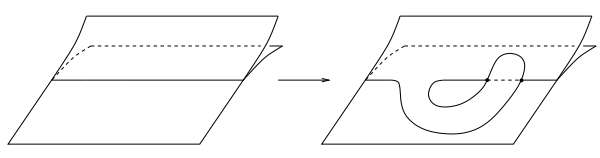

Figure 7: Another sliding move.

Standard sliding moves Let $P \rightarrow P^{\prime}$ be a positive MP-move (so, $P^{\prime}$ has one vertex and one region more than $P$ ). If $P$ has a branching, all the regions of $P^{\prime}$, except for the new one, already have an orientation, and it is a fact that the new region can always be given an orientation (sometimes not a unique one) so to get a branching on $P^{\prime}$. Each of the moves on branched spines arising like this will be called a branched MP-move, and it will be called a sliding MP-move if moreover it does not modify the boundary pattern of the associated concave field. One can actually see that each sliding-MP-move can be realized within a certain pair $(M, v)$ as a continuous deformation through branched spines of $M$ transverse to $v$, with only one singularity at which the spine is non-standard but transversality is preserved. This deformation is shown in Fig. 6, and it justifies the term 'sliding' quite clearly. Since in Fig. 6 we are showing portions of spines embedded in $\mathbb{R}^{3}$, to give a completely intrinsic description of the moves we should specify in each portion whether the screw-orientation of the spine is equal or opposite to that induced by $\mathbb{R}^{3}$, and whether the upward vertical field is positively or negatively transversal to the spine. As a result, the complete list of sliding-MP-moves contains 16 different ones, but the essential physical modifications are only those shown in Fig. 6. From this figure one also sees quite clearly that if $\widehat{v}(P)$ and $\widehat{v}\left(P^{\prime}\right)$ can be defined then they coincide (up to homotopy through fields compatible with $\widehat{\mathcal{P}}(P)=\widehat{\mathcal{P}}\left(P^{\prime}\right)$ ). Another move which obviously has the same property, and will be needed below, is the branched version of the 0-to-2 move, shown Fig. 0, and called the snake move in the sequel. As above, if one takes orientations into account, there is another essentially different snake move, obtained by mirroring Fig. 7. Since also the snake move involves a sliding, we will call standard sliding move any sliding-MP or snake move. 


\section{A calculus for combed manifolds with concave boundary}

In this section we will extend and refine the main results of Chapter 5 of [2]. The extension consists in passing from the closed to the bounded case, and the refinement comes from the shortening of the list of moves to be considered. More precisely, we will show that compact manifolds with concave combings are combinatorially described by (suitable) branched spines up to certain moves, namely the standard sliding (snake and sliding-MP) moves shown above. Moreover, we will show that spines which are rigid with respect to sliding-MP-moves can be dismissed with no harm, and that the slidingMP-moves suffice to generate the equivalence on the remaining spines. This implies that our result is a perfect combed analogue of the Matveev-Piergallini theorem (but our proof is self-contained).

Definitions and statements We will denote by Comb the set of all pairs $(M, v)$, where $M$ is a compact oriented manifold and $v$ is a concave field on $M$, viewed up to diffeomorphism of $M$ and homotopy of $v$ through concave fields. A class $[M, v] \in$ Comb is called a combing on the diffeomorphism class of the manifold $M$. Note that the boundary pattern on $\partial M$ evolves isotopically during a homotopy of $v$, so a pair $(M, \mathcal{P})$, viewed up to diffeomorphism of $M$, can be associated to each $[M, v] \in$ Comb. In particular, Comb naturally splits as the disjoint union of subsets $\operatorname{Comb}([M, \mathcal{P}])$, consisting of combings on $M$ compatible with $\mathcal{P}$.

For a technical reason we actually rule out from Comb the set of those classes $[M, v]$ such that the corresponding boundary pattern contains components of the type $S_{\text {triv }}^{2}$. This is actually not a serious restriction, because each $S_{\text {triv }}^{2}$ component can be capped off by a $B_{\text {triv }}^{3}$, and the result is well-defined up to homotopy. Note that we do accept pairs $(M, v)$ with closed $M$, and pairs in which $v$ has no tangency at all to $\partial M$.

Let us denote now by $\mathcal{B}$ the set of all branched spines $P$ (up to PL isomorphism) such that $\mathcal{P}(P)$ contains only one $S_{\text {triv }}^{2}$. Such a $P$ being given, $\widehat{M}(P)$ and $\widehat{v}(P)$ can be considered, and the pair $(\widehat{M}(P), \widehat{v}(P))$ gives rise to a well-defined element of Comb, which we denote by $C(P)$. The following will be shown below:

Theorem 2.1. The map $C: \mathcal{B} \rightarrow$ Comb is surjective, and the equivalence relation defined by $C$ on $\mathcal{B}$ is generated by sliding-MP-moves and snake moves.

Remark 2.2. The following interpretation of the surjectivity of $C$ is perhaps useful. Note first that the dynamics of a field, even a concave one, can be very complicated, whereas the dynamics of a traversing field (in particular, $B_{\text {triv }}^{3}$ ) is simple. Surjectivity of $C$ means that for any (complicated) concave field there exists a sphere $S^{2}$ which splits the field into two (simple) pieces: a standard $B_{\text {triv }}^{3}$ and a concave traversing field. 
Actually, a 1-parameter version of this statement also holds (see Remark 2.6): we will need it to show that the $C$-equivalence is the same as the sliding equivalence.

As announced, we state now the sliding analogue of the fact that the MP moves suffice. Let us denote by $\mathcal{R}$ the subset of $\mathcal{B}$ consisting of the branched spines which are "rigid" from the point of view of the sliding-MP-moves, i.e. the spines to which no such move applies. An explicit description of $\mathcal{R}$ is given in the proof of the next result. In the statement we only emphasize the most important consequences of this description.

Proposition 2.3. $\quad$ (i) For every surface $\Sigma$ and pattern $\mathcal{P}$ on $\Sigma$ there are at most two spines $P \in \mathcal{R}$ such that $\partial(M(P)) \cong(\Sigma, \mathcal{P})$.

(ii) If two elements of $\mathcal{B} \backslash \mathcal{R}$ are related through sliding-MP moves and snake moves, they are also related through sliding-MP-moves only.

(iii) Every $P \in \mathcal{R}$ is related by a snake move to an element of $\mathcal{B} \backslash \mathcal{R}$.

This proposition shows that in the statement of Theorem 2.1 one may remove $\mathcal{R}$ from $\mathcal{B}$ and forget the snake move.

Embedding-refined calculus We spell out in this paragraph the embedding-refined version of our calculus, which allows to neglect the action of automorphisms. Let a certain manifold $M$ be given, and consider the set $\operatorname{Comb}(M)$ of concave vector fields on $M$, up to homotopy. Let $\mathcal{B}(M)$ consist of the elements of $\mathcal{B}$ which are smoothly embedded in $M$ as spines of $M$ minus a ball $B_{\text {triv }}^{3}$. Each element $P$ of $\mathcal{B}(M)$ is viewed up to isotopy in $M$, and gives rise to a well-defined element $C_{M}(P)$ of $\operatorname{Comb}(M)$. Moreover sliding-MP-moves and snake moves are well-defined in $\mathcal{B}(M)$, because they can be realized as embedded moves. The embedded analogue of Theorem 2.1 states that $C_{M}: \mathcal{B}(M) \rightarrow \operatorname{Comb}(M)$ is surjective, and the relation it defines is generated by the embedded moves. The proof of this result is a refinement of the proof of the general statement, along the lines explained in [2] (4.1.12, 4.1.13, 4.3.5, and 5.2.1.)

Normal sections of a concave field The proof of Theorem 2.1 is an extension of the argument given in Chapter 5 of [2], and it is based on the following technical notion, which extends ideas originally due to Ishii [10]. Let $v$ be a concave field on $M$. Let $B_{1}, \ldots, B_{k}$ be the black components of the splitting of $\partial M$, i.e. the regions on which $v$ points outwards. A normal section for $(M, v)$ is a compact surface $\Sigma$ with boundary, embedded in the interior of $M$, with the following properties:

1. $v$ is transverse to $\Sigma$;

2. $\Sigma$ has exactly $k+1$ components $\Sigma_{0}, \ldots, \Sigma_{k}$, with $\Sigma_{0} \cong D^{2}$; 


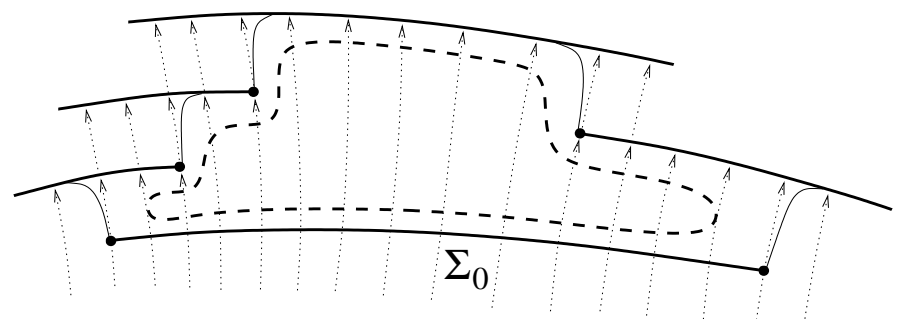

Figure 8: The trivial ball.

3. For $i>0$, the projection of $B_{i}$ on $\Sigma$ along the orbits of $-v$ is well-defined and yields a diffeomorphism between $B_{i}$ and a surface $B_{i}^{\prime}$ contained in the interior of $\Sigma_{i}$, with $\Sigma_{i} \backslash B_{i}^{\prime}$ being a collar on $\partial \Sigma_{i}\left(\right.$ so $B_{i}^{\prime}=\Sigma_{i}$ if $\left.\partial \Sigma_{i}=\emptyset\right)$;

4. Each positive half-orbit of $v$ meets either the interior of some $B_{i}$ (where it stops), or the interior of some $\Sigma_{i}$;

5. $\partial \Sigma$ meets itself generically along $v$ (i.e. each orbit of $v$ meets $\Sigma$ at most two consecutive times on $\partial \Sigma$, and, if so, transversely);

6. Let $P_{\Sigma}$ be the union of $\Sigma$ with all the orbit segments starting on $\partial \Sigma$ and ending on $\Sigma$. Then $\Sigma$, which is a simple polyhedron by the previous point, is actually standard.

The next two lemmas show that normal sections of $(M, v)$ correspond bijectively to spines $P$ such that $C(P)=[M, v]$. The proof of surjectivity of $C$ and the discussion of its non-injectivity will be based on these lemmas.

Lemma 2.4. If $(M, v), \Sigma$ and $P_{\Sigma}$ are as above, then $P_{\Sigma}$ can be given a structure of branched spine such that $C\left(\left[P_{\Sigma}\right]\right)=[M, v]$.

Proof of 2.4. We orient $\Sigma$ so that $v \pitchfork^{+} \Sigma$ (by default $M$ is oriented). Every region of $P_{\Sigma}$ contains some open portion of $\Sigma$, so it can be oriented accordingly; with the obvious screw-orientation, this turns $P_{\Sigma}$ into a branched spine of its regular neighbourhood in $M$.

We show that $C\left(\left[P_{\Sigma}\right]\right)=[M, v]$ by embedding the abstract manifold $M\left(P_{\Sigma}\right)$ in $M$, in such a way that the field carried by $P_{\Sigma}$ on $M\left(P_{\Sigma}\right) \subset M$ is just the restriction of $v$. By construction, $M \backslash M\left(P_{\Sigma}\right)$ will consist of a copy of $B_{\text {triv }}^{3}$, together with a collar on $\partial M$ which can be parameterized as $(\partial M) \times[0,1]$ in such a way that $v$ is constant in the $[0,1]$-direction. This easily implies that $C\left(\left[P_{\Sigma}\right]\right)=[M, v]$ indeed.

We illustrate the embedding of $M\left(P_{\Sigma}\right)$ in $M$ pictorially in one dimension less. Figure 8 shows how $\Sigma_{0}$ gives rise to a $B_{\text {triv }}^{3}$. In the figure we describe $v$ by dotted lines, $\Sigma$ by thick lines, portions of $P_{\Sigma} \backslash \Sigma$ by thin lines, and $\partial\left(M\left(P_{\Sigma}\right)\right)$ by a thick dashed 




Figure 9: Collar on a boundary component.

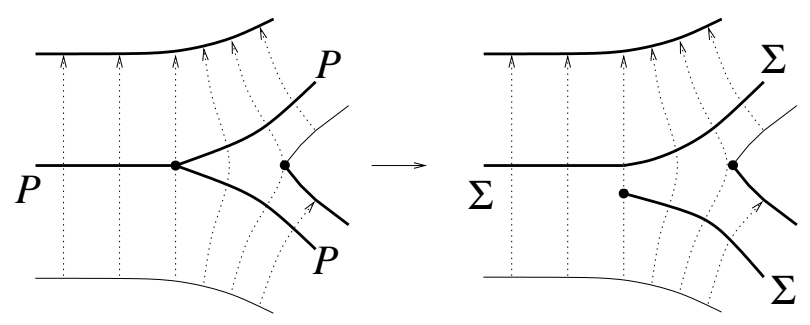

Figure 10: Normal section from a spine.

line. Note also that the portions of $P_{\Sigma} \backslash \Sigma$ have been slightly modified so to become positively transversal to $v$, which allows us to represent the branching as usual, i.e. as a $\mathrm{C}^{1}$ structure on $P_{\Sigma}$.

Figure 9 shows the collar based on a component of $\partial M$. We use the same conventions as in the previous figure, and in addition we represent the black and white components of $\partial M$ by thick and thin lines respectively. This description concludes the proof.

Lemma 2.5. Let $[P] \in \mathcal{B}$ and $C([P])=[M, v] \in$ Comb, with $P$ embedded in $(M, v)$ according to the geometric description of $C$. Let $\Sigma$ be obtained from $P$ as suggested (in one dimension less) in Fig. 10. Then $\Sigma$ is a normal section of $(M, v)$, and $P_{\Sigma}$ is isomorphic to $\Sigma$.

Proof of 2.5. The construction suggested by Fig. 10 is obviously the inverse of the construction in the proof of Lemma 2.4. 
The concave combing calculus Using normal sections we can now show the main result of this section.

Proof of 2.1. We start with the proof of surjectivity. So, let us consider a combed manifold $(M, v)$, subject to the usual restrictions. By Lemma 2.4 it is natural to try and construct a normal section for $(M, v)$. Let $B_{1}, \ldots, B_{k}$ be the black regions in $\partial M$. Slightly translate each $B_{i}$ along $-v$, getting $B_{i}^{\prime}$. Add to each $B_{i}^{\prime}$ a small collar normal to $v$, getting $\Sigma_{i}$ (if $\partial B_{i}=\emptyset$, we set $\Sigma_{i}=B_{i}^{\prime}$ ). Select finitely many discs $\left\{D_{n}\right\}$ disjoint from each other and from all the $\Sigma_{i}$ 's, such that all positive orbits of $v$, except for the small segments between $B_{i}^{\prime}$ and $B_{i}$, meet $\left(\bigcup_{i \geq 1} \Sigma_{i}\right) \cup\left(\cup D_{n}\right)$ in some interior point. Connect the $D_{n}$ 's together by strips transversal to $v$ and disjoint from $\bigcup_{i \geq 1} \Sigma_{i}$, getting a disc $\Sigma_{0}$. Up to a generic small perturbation, the surface $\Sigma=\bigcup_{i \geq 0} \Sigma_{i}$ satisfies all axioms of a normal section for $(M, v)$, except axiom 6 .

Now, even if it is not standard, $P_{\Sigma}$ can be defined, and the proof of Lemma 2.4 shows that it is a simple branched spine of $\left(M \backslash B^{3}, v\right)$. In particular, $P_{\Sigma}$ is connected and its singular locus is non-empty. We recall now that in Chapter 4 of [2] we have considered a set of local moves on simple branched spines, called 'simple sliding moves', which preserve the transversal field (and hence the splitting of the boundary), but do not require or preserve the cellularity condition. Knowing that $P_{\Sigma}$ is connected and $S\left(P_{\Sigma}\right) \neq \emptyset$, it is not too hard to see that there exists a sequence of (abstract) simple sliding moves which turns $P_{\Sigma}$ into a standard spine (see [2], Section 4.4). If we physically realize these moves within $M$, preserving transversality to $v$, the result is a standard branched spine $P$ such that $C([P])=[M, v]$.

We are left to show that if $C\left(\left[P_{0}\right]\right)=C\left(\left[P_{1}\right]\right)$ then $P_{0}$ and $P_{1}$ are related by slidingMP-moves and snake moves ('sliding-equivalent' for short). By the definition of Comb and $C$, using also the above lemmas, there exists a manifold $M$ and a homotopy $\left(v_{t}\right)$ of concave fields on $M$, such that $P_{0}$ and $P_{1}$ are defined by normal sections $\Sigma^{(0)}$ and $\Sigma^{(1)}$ of $\left(M, v_{0}\right)$ and $\left(M, v_{1}\right)$ respectively.

We prove that $P_{0}$ and $P_{1}$ are sliding-equivalent first in the special case where $v_{0}=$ $v_{1}=v$. The general case will be an easy consequence. For $j=0,1$, let $\Sigma^{(j)}=\bigcup_{i>0} \Sigma_{i}^{(j)}$. Proceeding as in the above proof of surjectivity, for each black region $B_{i}$ of $\partial M$, we consider a collared negative translate $\bar{\Sigma}_{i}$ of $B_{i}$. We choose $\bar{\Sigma}_{i}$ so close to $B_{i}$ that $\bar{\Sigma}_{i} \cap \Sigma^{(j)}=\emptyset$, and the negative integration of $v$ yields a diffeomorphism from $\bar{\Sigma}_{i}$ to a subset of $\Sigma_{i}^{(j)}$.

SteP I. For $j=0,1$, there exists a disc $D_{j}$ such that $D_{j} \cup\left(\bigcup_{i \geq 1} \bar{\Sigma}_{i}\right)$ is a normal section of $(M, v)$, and the associated branched spine is sliding-equivalent to $P_{j}$. To prove this, we temporarily drop the index $j$. We first isotope each $\Sigma_{i}$, without changing the associated spine, until it contains $\bar{\Sigma}_{i}$, as suggested in Fig. 11 .

Note that if $\partial B_{i}=\emptyset$ we automatically have $\Sigma_{i}=\bar{\Sigma}_{i}$. Otherwise, we concentrate on one of the annuli $A$ of which $\Sigma_{i} \backslash \bar{\Sigma}_{i}$ consists. Note that we cannot just shrink $A$ leaving the rest of the section unchanged, because we could spoil axiom 14 of the definition of normal section. To actually shrink $A$ we first need to "insulate" it, toward the positive 


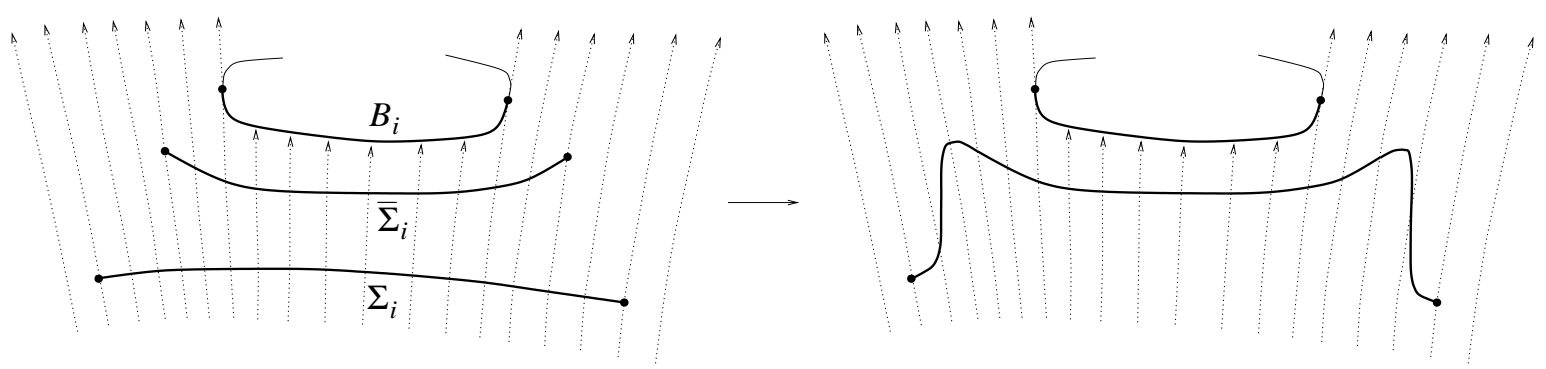

Figure 11: Isotopy of a normal section.
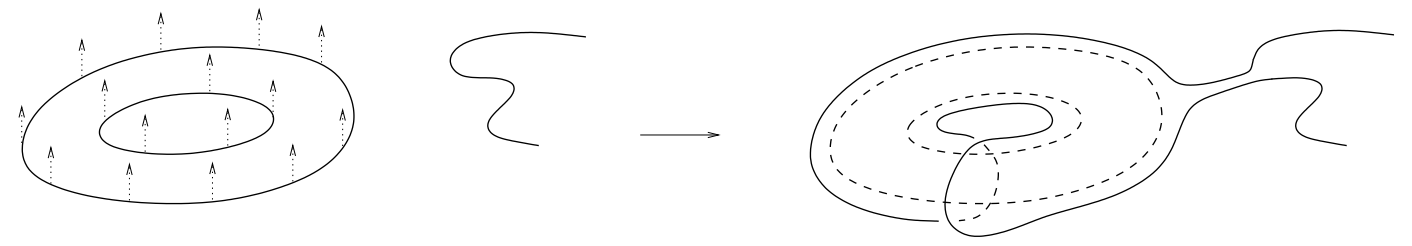

Figure 12: Insulation of an annulus.

direction of $v$, by adding to the disc $\Sigma_{0}$ a strip normal to $v$. Figure 12 suggests how to do this.

As we modify $\Sigma_{0}$ as suggested, it is clear that we keep having a "quasi-normal" section, i.e. all axioms except 6 hold. Moreover the corresponding simple branched spines are obtained from each other by the simple sliding moves already mentioned above. To conclude we apply, as above, the fact that a simple branched spine can be transformed via simple sliding moves to a standard one, and the technical result established in [2], Proposition 4.5.6, according to which standard spines which are equivalent under simple sliding moves are also sliding-equivalent. This proves Step I.

The conclusion will now follow quite closely the argument in [2], Theorem 5.2.1.

STEP II. There exist discs $D_{0}^{\prime}$ and $D_{1}^{\prime}$ such that $D_{j}^{\prime} \cup\left(\cup_{i \geq 1} \bar{\Sigma}_{i}\right)$ is a normal section of $(M, v)$ for $j=0,1$, and $D_{0} \cap D_{0}^{\prime}=D_{0}^{\prime} \cap D_{1}^{\prime}=D_{1}^{\prime} \cap D_{1}=\emptyset$. Choosing a metric on $M$, one can construct $D_{0}^{\prime}$ and $D_{1}^{\prime}$ by first taking many very small discs almost orthogonal to $v$, and then connecting these discs by strips transversal to $v$.

STEP III. Conclusion in the case $v_{0}=v_{1}$. If we connect $D_{0}$ and $D_{0}^{\prime}$ by a strip orthogonal to $v$, we get a bigger disc $\tilde{D}_{0}$ such that $\tilde{D}_{0} \cup\left(\bigcup_{i \geq 1} \bar{\Sigma}_{i}\right)$ is still a normal section of $(M, v)$. We can actually imagine a dynamical process, in which $D_{0}$ is first enlarged to $\tilde{D}_{0}$, and then is reduced to $D_{0}^{\prime}$, as in Fig. 13. If the transformation is chosen generic enough, at all times axioms 1, 2, 囵 and t will hold, and axiom 5 will hold at all but finitely many times. This means that the corresponding branched spines are related by simple sliding moves. Similarly, we can replace $D_{0}^{\prime}$ first by $D_{1}^{\prime}$ and then by $D_{1}$. Using the facts quoted above, the conclusion follows.

We are left to deal with the general case, where $\left(v_{t}\right)$ is a non-constant homotopy. It 


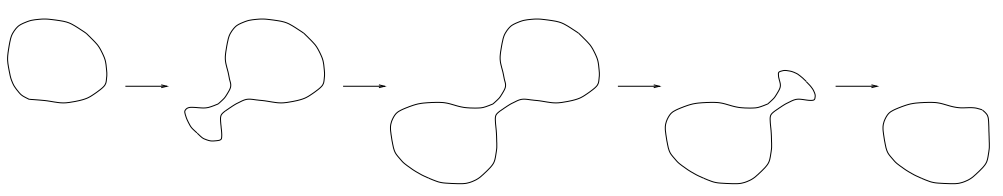

Figure 13: Transformation of a disc into a disjoint one.

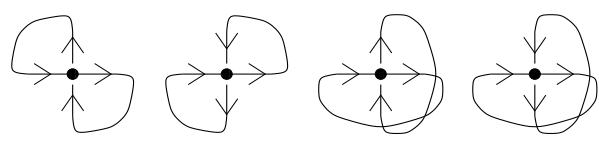

Figure 14: Branched spines with one vertex.

is then sufficient to take a partition $0=t_{0}<t_{1}<\ldots<t_{n}=1$ of $[0,1]$, fine enough that $\left(M, v_{t_{k-1}}\right)$ and $\left(M, v_{t_{k}}\right)$ admit a common normal section which gives rise to isomorphic branched spines.

Remark 2.6. Along the lines of the previous proof we have established the following topological fact, whose statement does not involve spines. Let $\left(v_{t}\right)$ be a homotopy of concave fields on $M$, let $B_{0}, B_{1} \subset M$ be balls with $\left(B_{j}, v_{j}\right) \cong B_{\text {triv }}^{3}$ and $v_{j}$ traversing on $M \backslash B_{j}$ for $j=0,1$. Then there exist another homotopy $\left(v_{t}^{\prime}\right)$ between $v_{0}$ and $v_{1}$ and an isotopy $\left(B_{t}\right)$ with $\left(B_{t}, v_{t}\right) \cong B_{\text {triv }}^{3}$ and $v_{t}$ traversing on $M \backslash B_{t}$ for all $t$.

Sufficiency of the sliding-MP-moves To show Proposition 2.3 we will find it convenient to use the graphic representation of branched spines introduced in [2], Section 3.2 , but we do not reproduce here the technicalities needed to introduce this representation.

Proof of 2.3. We start by listing rigid spines. Note first that if a negative slidingMP-move applies to a spine then also a positive one does, so we only need to consider positive rigidity. The spines with one vertex, shown in Fig. 14, are of course rigid. Using [2], Proposition 3.3.5, one easily checks that $\partial(M(P))$ is $S_{\text {triv }}^{2}$ for the first two spines, and $S_{\text {triv }}^{2} \sqcup S_{\text {triv }}^{2}$ for the other two.

Now we turn to rigid spines with more than one vertex. Rigidity implies that all edges with distinct endpoints should appear as on the left in Fig. 15. It is not hard to deduce that rigid spines come in a sequence $P_{1}^{\text {rig }}, P_{2}^{\text {rig }}, \ldots$ as shown in the rest of Fig. 15, where $P_{k}^{\text {rig }}$ has $2 k$ vertices, and $\partial\left(M\left(P_{k}^{\text {rig }}\right)\right)$ is the union of $S_{\text {triv }}^{2}$ together with



To show (ii) we must prove that:

(ii-a) Sequences which contain rigid spines can be replaced by sequences which do not.

(ii-b) If two non-rigid spines are related by one snake move then they are also related by a sequence of sliding-MP-moves. 

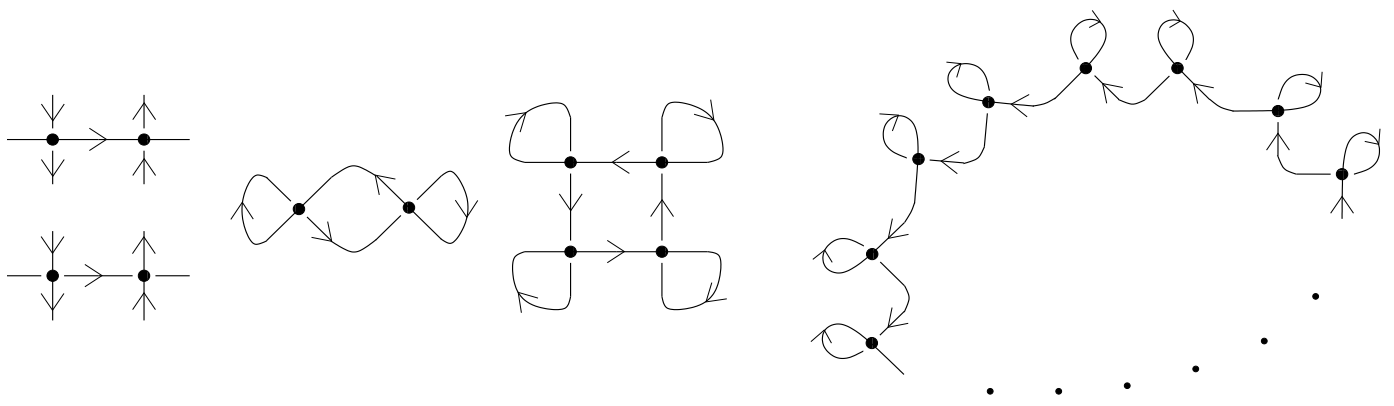

Figure 15: Sliding-MP-rigid branched spines.

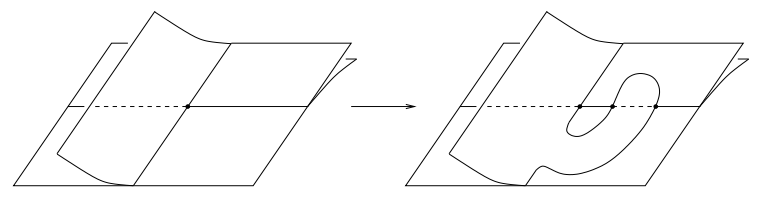

Figure 16: The sliding-vertex move.

For (ii-a), we note that the result of a positive snake move is never rigid. So if a rigid spine $P$ appears in a sequence of moves then $P$ is the result of a negative snake move $\mu_{1}^{-1}: P_{1} \rightarrow P$, and a positive snake move $\mu_{2}: P \rightarrow P_{2}$ is applied to $P$. Since all edges of a spine survive through a snake move, there is a version $\tilde{\mu}_{2}$ of $\mu_{2}$ which applies to $P_{1}$ and a version $\tilde{\mu}_{1}$ of $\mu_{1}$ which applies to $P_{2}$, and the result $\tilde{P}$ is the same. So can replace the segment $\left(P_{1}, P, P_{2}\right)$ by $\left(P_{1}, \tilde{P}, P_{2}\right)$, and now all the spines involved are non-rigid.

Let us turn to (ii-b). The proof results from three steps, to describe which we introduce in Figure 16 another move, called sliding-vertex move, whose unbranched version was already considered in [11] and [13]. Again, taking into account orientations, there are two versions of the move (for each vertex type), but we will ignore this detail.

Step 1: if $v$ is a vertex of a branched spine $P, e$ is any one of the edges incident to $v, P_{v}$ is obtained from $P$ via the sliding-vertex move at $v$, and $P_{e}$ is obtained from $P$ via the snake move on $e$, then $P_{v}$ and $P_{e}$ are related by sliding-MP-moves. This is proved by an easy case-by-case analysis. It turns out that two MP-moves (a positive and a negative one) are always sufficient.

Step 2: let $v, P$ and $P_{v}$ be as above. If $P$ and $P_{v}$ are related by sliding-MP-moves, the same is true for $P$ and any spine obtained from $P$ by a snake move. To see this, use step 1 to successively transform sliding-vertex moves into snake moves and conversely, until the desired snake move is reached.

Step 3: if $P$ is non-rigid then there exists a vertex $v$ such that $P$ and $P_{v}$ are related by MP-moves. The vertex $v$ is chosen to be an endpoint of an edge to which the positive MP-move applies. The argument is again a long case-by-case one, which refines in a branched context the argument given by Piergallini in [13]. The sequence 


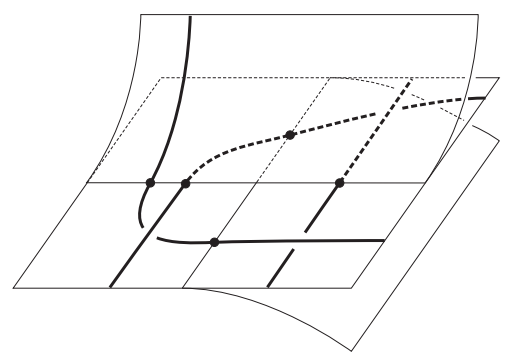

Figure 17: A portion of $\mathrm{C}^{1}$ link diagram on a branched spine.

always consists of three positive moves followed by a negative one. This concludes the proof of (ii), whereas (iii) is evident.

2.3

\section{A calculus for framed links}

We fix in this section a compact manifold $M$ and consider the set $\operatorname{Fram}(M)$ of isotopy classes of framed links in $M$. Since a link isotopy generically avoids a fixed 3-ball, $\operatorname{Fram}(M)$ and $\operatorname{Fram}(\widehat{M})$ are canonically isomorphic when $\partial M=S^{2}$, so we can restrict to non-closed $M$ 's and include the closed case as usual.

Statement Let us fix a branched standard spine $P$ of $M$. The fact that such a spine always exists was proved as Theorem 3.4.9 in [2]. We call $\mathrm{C}^{1}$ link diagram on $P$ an immersion of a disjoint union of circles into $P$, with generic intersection with $S(P)$ appearing as in Fig. 17, generic self-intersections (crossings), and the usual underover marking at crossings (as shown in the same figure; here 'under' and 'over' refer to the field positively transversal to $P$ ). The set of all $\mathrm{C}^{1}$ link diagrams on $P$ will be denoted by $\mathcal{D}(P)$. An element of $\mathcal{D}(P)$ obviously defines a link. Moreover $v(P)$ is transversal to this link, so it defines a framing, and we get an (obviously welldefined) map $F_{(P, M)}: \mathcal{D}(P) \rightarrow \operatorname{Fram}(M)$. Besides isotopy on $P$ through immersions having the same configuration of crossings and intersections with $S(P)$, there are several combinatorial moves which of course do not modify the isotopy class of the framed link defined by a diagram. We show a list of moves having this property in Fig. 18. For a reason to be given below, which also explains the apparently weird notation, we call these moves $\mathrm{C}^{1}$-Turaev moves. As we did when we described the sliding-MP-moves in Fig. 6, we are showing in Fig. 18 only the essential physical modifications, without specifying the screw-orientation of the spine and the orientation of its regions.

Theorem 3.1. The map $F_{(P, M)}: \mathcal{D}(P) \rightarrow \operatorname{Fram}(M)$ is surjective, and the equivalence relation it defines is generated by $\mathrm{C}^{1}$-Turaev moves.

Our argument, after the easy proof of surjectivity, goes along the following lines: 

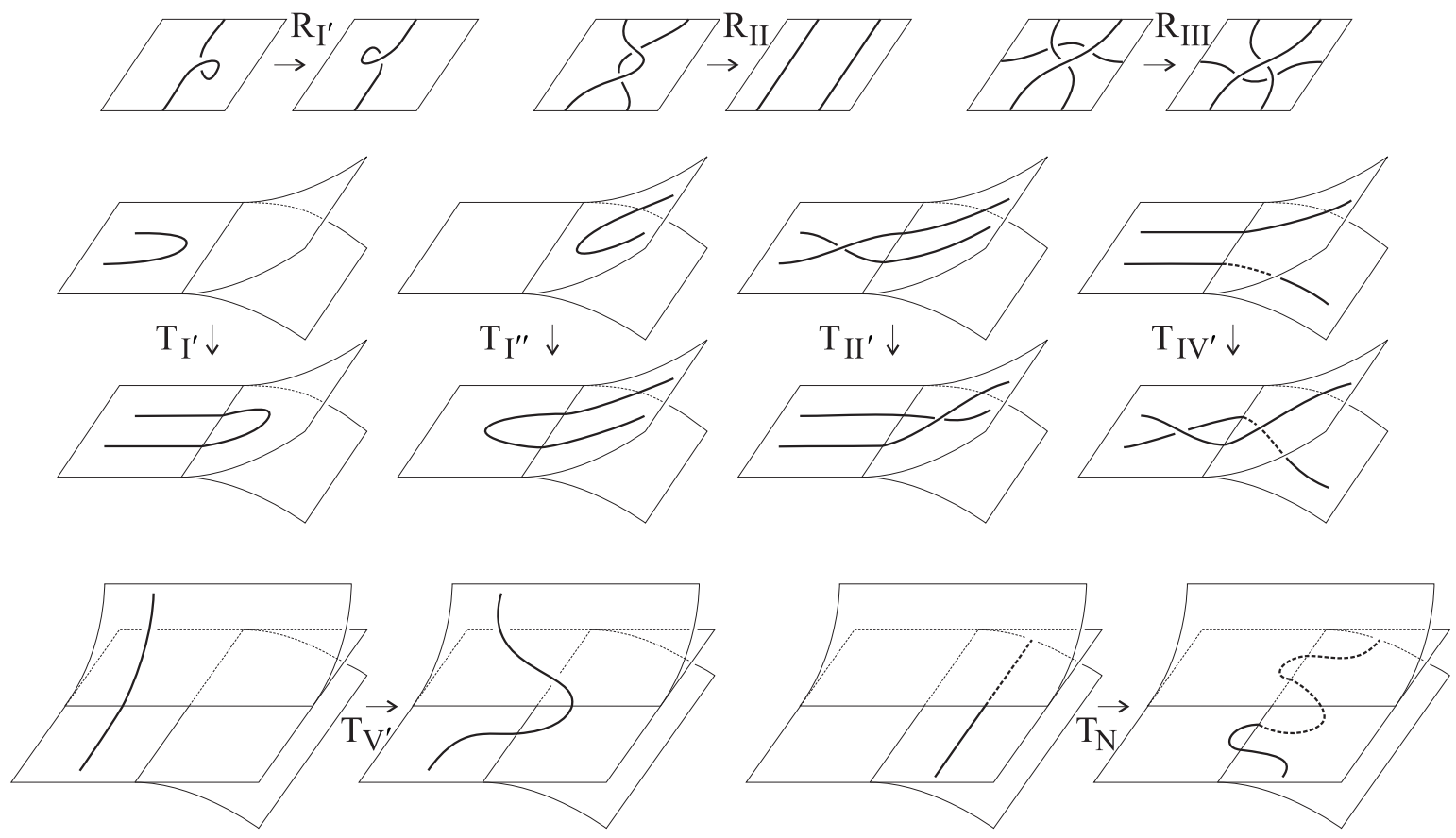

Figure 18: $\mathrm{C}^{1}$-Turaev moves.

1. We state the analogue of Theorem 3.1 for non-branched spines, due to Turaev [16] (we include a quick proof for the sake of completeness);

2. We modify Turaev's result to the case of a branched spine, but allowing non- $\mathrm{C}^{1}$ diagrams;

3. We prove our theorem, showing how to canonically replace each non- $\mathrm{C}^{1}$ diagram by a $\mathrm{C}^{1}$ one along a sequence of modifications.

Surjectivity Since $M$ and $P$ are fixed, we write $F$ for short. Given a framed link $L$ in $M$, we can prove that it is contained in the image of $F$ as follows:

- First, forget the framing and take a generic projection on $P$, recalling that $M \backslash$ $P \cong \partial M \times(0,1]$

- Next, eliminate non- $\mathrm{C}^{1}$ intersections with $S(P)$ as shown in Fig. 19 (left).

- Finally, give the resulting projection the right framing by adding the necessary numbers of curls. (Here we use the fact that two framings on a given knot differ at most by a finite number of full rotations.)

It may be noted that surjectivity of $F$ is preserved by restriction to the set of diagrams without crossings. This follows quite easily from the fact that all the regions 

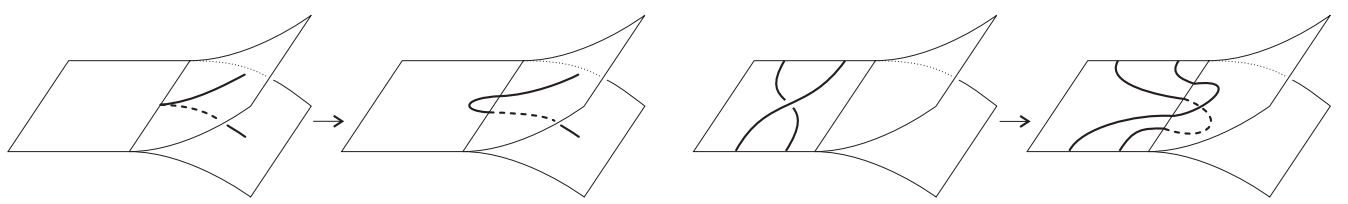

Figure 19: How to remove forbidden intersections with $S(P)$ and crossings.
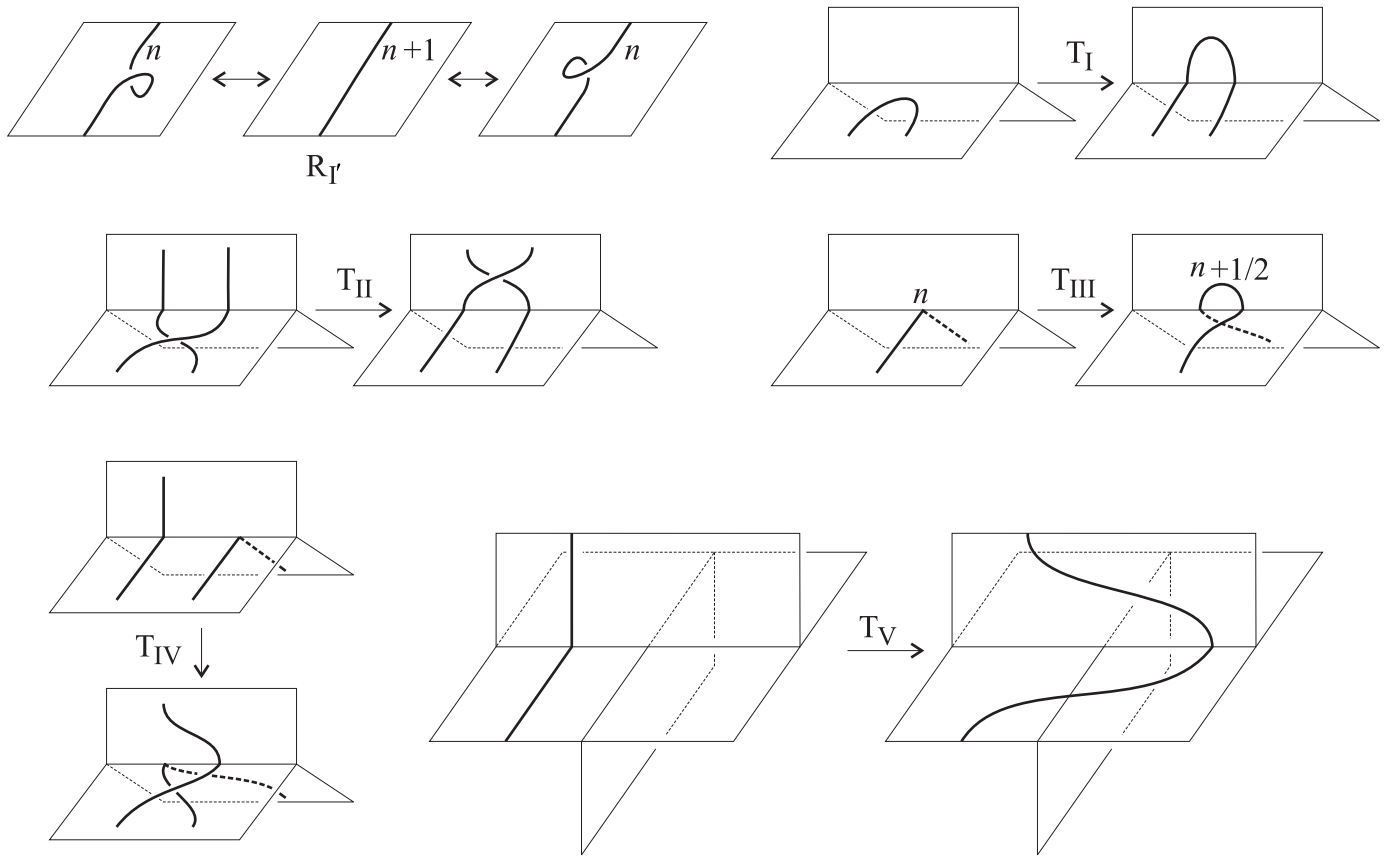

Figure 20: Turaev moves.

of $P$ have non-empty boundary, as suggested in Fig. 19 (right). This property will not be used below.

Turaev moves on standard spines The ideas and results of this paragraph are due to Turaev [16]. We temporarily allow $P$ to be any standard spine of $M$, not a branched one. If each region of $P$ is given an arbitrary transverse orientation, the definition of a link diagram $D$ makes sense also on $P$, but $D$ may not define a framing on the associated link, because the strip which runs along a component of $D$ on $P$ need not be a cylinder, it may be a Möbius strip. So we attach to each component $D_{i}$ of $D$ a full or half-integer $a_{i} / 2$, depending on the topology of the strip, and we define the framing by giving $a_{i}$ positive half-twists to the strip (recall that $M$ is oriented). By diagram on $P$ we will actually mean one such pair $\left(\left\{D_{i}\right\},\left\{a_{i} / 2\right\}\right)$.

We call Turaev moves those shown in Fig. 20, together with the $R_{\mathbb{I I}}$ and $R_{\text {II }}$ already shown above. In Fig. 20, for $\mathrm{R}_{\mathrm{I}^{\prime}}$ and $\mathrm{T}_{\mathrm{III}}$, the local orientation must be that of $\mathbb{R}^{3}$. 


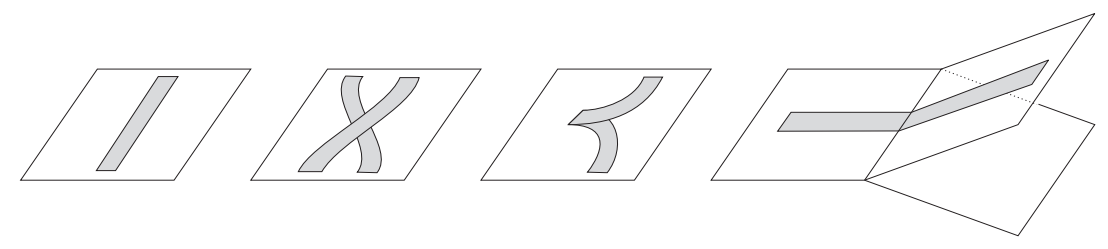

Figure 21: Projection of a strip.

Theorem 3.2. Every isotopy class of framed link in $M$ is defined by some diagram on $P$, and two diagrams define the same class if and only if they are obtained from each other by a sequence of Turaev moves.

Proof of 3.2. Recall that a framed link can be thought of as an embedded cylinder. Moreover $M$ projects onto $P$, and the projection of a cylinder generically appears as in Fig. 21. Such a projection easily defines a diagram, the half-integers being sums of $\pm 1 / 2$ 's corresponding to the bends of the projection. Moreover, the elementary catastrophes along an isotopy of a projection translate into the moves of Fig. 20, or simple combinations of them.

Turaev moves on branched spines Going back to the case where $P$ is branched, we can still apply Theorem 3.2, but now the list of moves becomes slightly longer, if we want to take the branching into account.

Proposition 3.3. If $P$ is a branched spine then any Turaev move for a diagram on $P$ can be expressed as a combination (including inverses) of the moves $\mathrm{R}_{\mathrm{I}^{\prime}}, \mathrm{R}_{\mathbb{I}}, \mathrm{R}_{\mathbb{I I}}$, $\mathrm{T}_{\mathrm{I}^{\prime}}, \mathrm{T}_{\mathrm{I}^{\prime \prime}}, \mathrm{T}_{\mathbb{I}^{\prime}}, \mathrm{T}_{\mathrm{I}^{\prime}}, \mathrm{T}_{\mathrm{V}^{\prime}}$ shown above, together with the moves $\mathrm{T}_{\mathrm{I}^{\prime \prime \prime}}, \mathrm{T}_{\mathbb{I}^{\prime \prime}}, \mathrm{T}_{\mathbb{I I}^{\prime}}, \mathrm{T}_{\mathbb{I}^{\prime \prime}}, \mathrm{T}_{\mathrm{V}^{\prime \prime}}$ shown in Fig. 29

Proof of 3.3. The branching can be interpreted as a loss of symmetry of a spine, so each of Turaev's moves, when viewed as a move on a branched spine, generates many different ones according to the position of the diagram with respect to the branching. The result is a list much longer than that given in the statement, but one can show that all the moves omitted from the statement are generated by the moves included. Two examples are provided in Figg. 23 and 24.

$\mathbf{C}^{\mathbf{1}}$ moves We can now conclude the proof of Theorem 3.1 (surjectivity having been shown above). We are left to show that if $D$ and $D^{\prime}$ are $\mathrm{C}^{1}$-diagrams on $P$ which define the same framed link, then they are related by a sequence of $\mathrm{C}^{1}$ Turaev moves. By Theorem 3.2 and Proposition 3.3, there exists a sequence $D=D_{0} \rightarrow D_{1} \rightarrow \cdots \rightarrow$ $D_{n-1} \rightarrow D_{n}=D^{\prime}$ where each move $D_{i-i} \rightarrow D_{i}$ is one of those listed in Proposition 3.3 . 

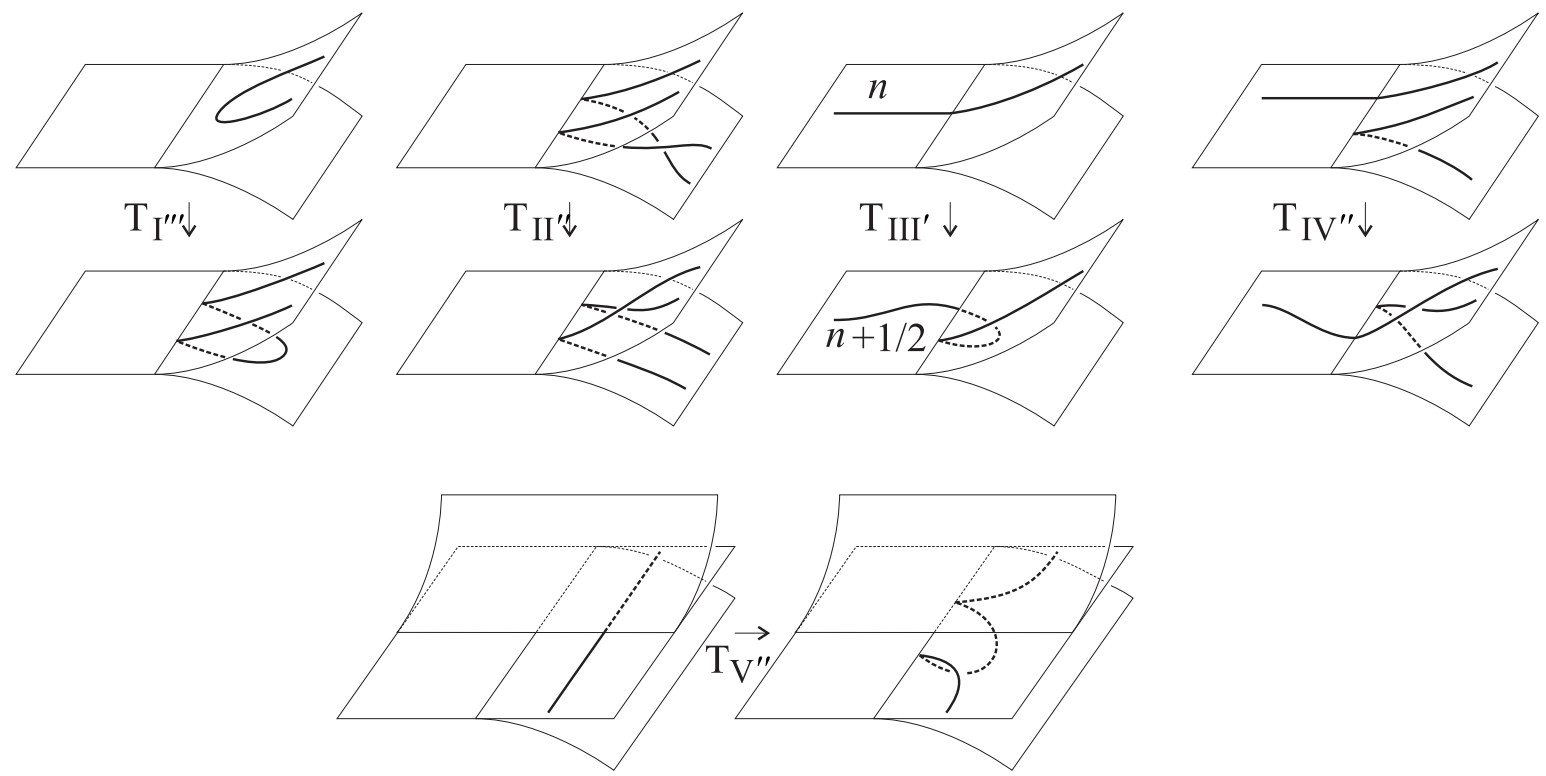

Figure 22: Non-C $\mathrm{C}^{1}$ Turaev moves on branched spines.

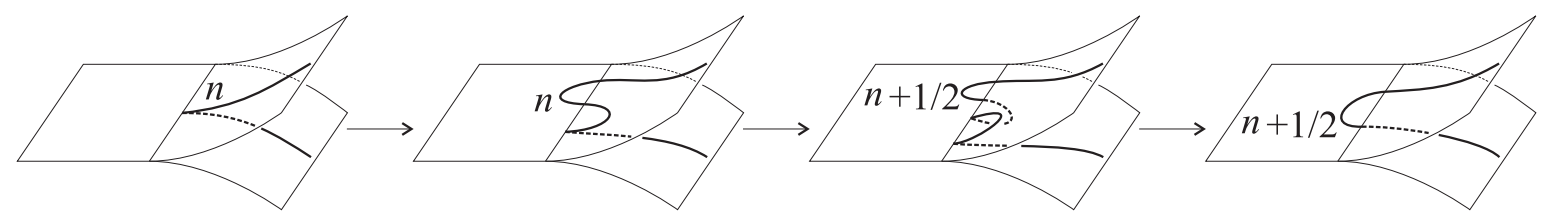

Figure 23: A sequence of moves $\mathrm{T}_{\mathrm{I}^{\prime \prime}}, \mathrm{T}_{\mathbb{I I}^{\prime}}$ and $\mathrm{T}_{\mathrm{I}^{\prime \prime \prime}}$ generating a branched version of $\mathrm{T}_{\mathrm{III}}$ omitted from the statement.



Figure 24: Another generation of moves, involving $\mathrm{T}_{\mathrm{I}^{\prime}}, \mathrm{T}_{\mathrm{V}^{\prime \prime}}$ and $\mathrm{T}_{\mathrm{I}^{\prime \prime \prime}}$. 
In particular the $D_{i}$ 's with $0<i<n$ can be non- $\mathrm{C}^{1}$ and can have a non-zero halfinteger attached to them. We will now show how to construct a modified sequence $D=\tilde{D}_{0} \rightarrow \tilde{D}_{1} \rightarrow \cdots \rightarrow \tilde{D}_{n-1} \rightarrow \tilde{D}_{n}$ with the following properties:

1. each $\tilde{D}_{i}$ is a $\mathrm{C}^{1}$ diagram with number 0 attached;

2. each $\tilde{D}_{i}$ is obtained from $\tilde{D}_{i-1}$ by a sequence of $\mathrm{C}^{1}$ Turaev moves;

3. each $\tilde{D}_{i}$ differs from $D_{i}$ for the presence of some extra curls; in particular each component $\tilde{D}_{i}^{(j)}$ of $\tilde{D}_{i}$ has a natural companion $D_{i}^{(j)}$ in $D_{i}$, with the property that, as unframed knots, the knots associated to $\tilde{D}_{i}^{(j)}$ and $D_{i}^{(j)}$ are both contained in a solid torus $T_{i}^{(j)}$ and parallel to the core of the torus;

4. the framed knots associated to $\tilde{D}_{i}^{(j)}$ and $D_{i}^{(j)}$ are framed-isotopic within $T^{(j)}$.

Requirement 4 is the crucial technical point of our proof. To verify that the requirement is stronger than just framed isotopy, note that in $D^{2} \times S^{1}$ the framings on the core $\{0\} \times S^{1}$ are parameterized by the integers, but, when $D^{2} \times S^{1}$ is mirrored in its boundary to get $S^{2} \times S^{1}$, only two inequivalent framings remain (corresponding to even and odd integers).

We assume for a moment the sequence $\tilde{D}_{i}$ to exist, and we show how to conclude. The transformation from $D$ to $\tilde{D}_{n}$ is made with $\mathrm{C}^{1}$ Turaev moves, so we only need to compare $\tilde{D}_{n}$ and $D^{\prime}=D_{n}$, which by assumption differ for some curls. Using $\mathrm{C}^{1}$ Turaev moves we can easily make all these curls slide until they are consecutive on the diagram. We recall now that there are four local pictures for a curl, depending on its local contributions \pm to the framing and to the winding number [15]. Assumption 4 now implies that the algebraic sum of local contributions to the framing vanishes. Therefore we can cancel the curls in pairs, either by moves $R_{I^{\prime}}$ (when the local contributions to the winding number are the same), or by a combination of moves $R_{\mathbb{I I}}$ and $R_{\mathbb{I I}}$ (when the contributions cancel). This shows the conclusion.

We are left to define the sequence $\tilde{D}_{i}$. The idea is simply not to perform the moves which change the half-integer or introduce cusps, and show that the sequence of moves can be followed anyway. While doing this we need to keep track of the portions where the new diagram $\tilde{D}_{i}$ differs from $D_{i}$, which we do by marking a neighbourhood of the portion as a shadowed box.

The moves which change the colour or introduce cusps are $\mathrm{R}_{\mathrm{I}^{\prime}}$ (in both directions), $\mathrm{T}_{\mathrm{I}^{\prime \prime \prime}}, \mathrm{T}_{\mathbb{I I}^{\prime}}$, and $\mathrm{T}_{\mathrm{V}^{\prime \prime}}$, and we show in Figg. 25 and 26 what we replace them with. For move $\mathrm{R}_{\mathrm{I}^{\prime}}$ it has been necessary to be more specific because, in the original definition of the move, two different ones were actually defined at the same time.

To show that $\left\{\tilde{D}_{i}\right\}$ can indeed be constructed we must now show that after performing the construction up to some level $k$ we can still still follow the rest of the sequence and go on with the replacements of Figg. 25 and 26. By construction $\tilde{D}_{k}$ differs from $D_{k}$ only within some shadowed boxes. We denote by $\mu_{k}$ the move $D_{k} \rightarrow D_{k+1}$ and 

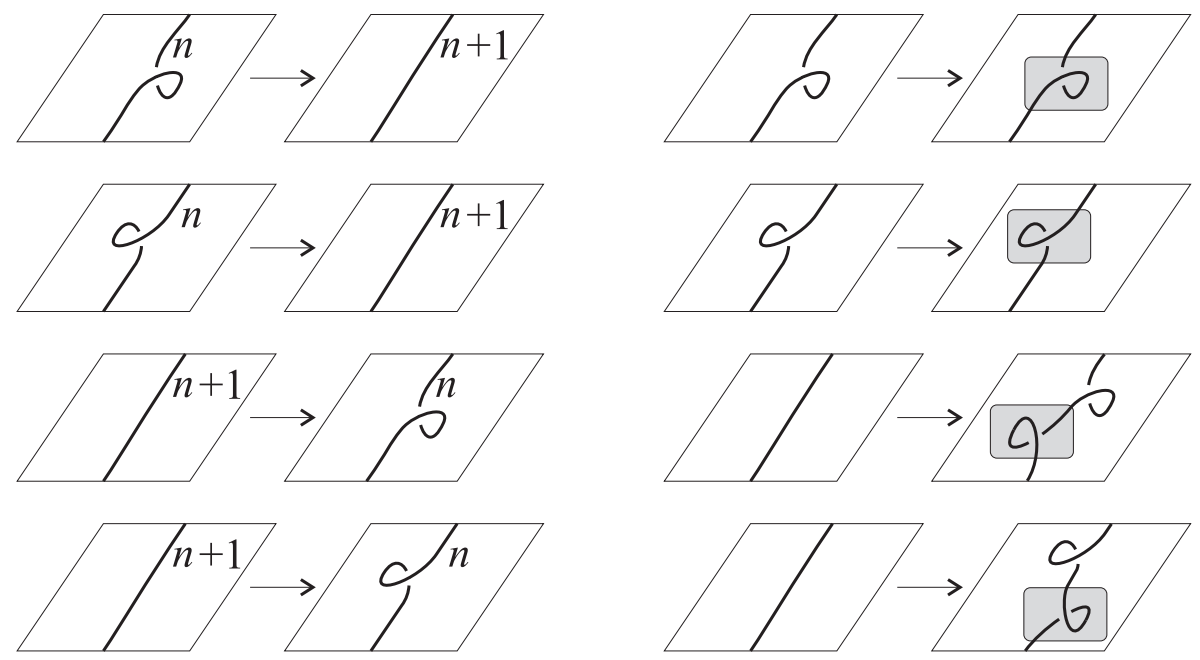

Figure 25: The moves $\mathrm{R}_{\mathrm{I}}$ (left), and the $\mathrm{C}^{1}$ moves replacing them (right).
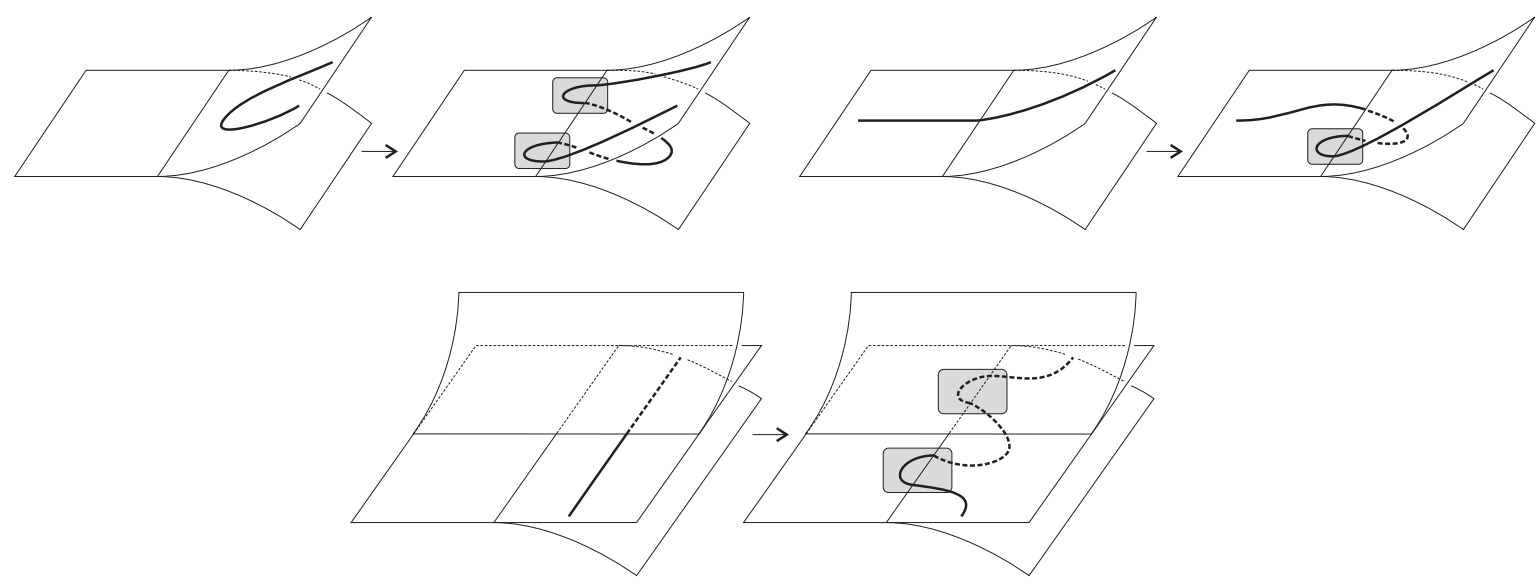

Figure 26: Replacement of the other non- $\mathrm{C}^{1}$ moves $\mathrm{T}_{\mathrm{I}^{\prime \prime \prime}}, \mathrm{T}_{\mathbb{I I}^{\prime}}$ and $\mathrm{T}_{\mathrm{V}^{\prime \prime}}$ by $\mathrm{C}^{1}$ moves. 
explain how to lift it to a move on $\tilde{D}_{k}$. First, note that a shadowed box lying within a region of $P$ and containing a curl does not interfere with $\mu_{k}$ whatever its type (but it may be necessary to add some $R_{\mathbb{I}}$ 's and $R_{\text {III }}$ 's to replace isotopy supported within the region).

We fix now our attention on a shadowed box $B$ which lies on $S(P)$ and contains a smoothed cusp, and examine the various instances for $\mu_{k}$ with respect to $B$. If $\mu_{k}$ is of type $\mathrm{R}_{\mathrm{I}}^{ \pm 1}, \mathrm{R}_{\mathrm{II}}^{ \pm 1}, \mathrm{~T}_{\mathrm{I}^{\prime}}^{ \pm 1}, \mathrm{~T}_{\mathrm{I}^{\prime \prime}}^{ \pm 1} \mathrm{~T}_{\mathbb{I}^{\prime}}^{ \pm 1}$, or $\mathrm{T}_{\mathrm{I}^{\prime}}^{ \pm 1}$, then it obviously does not interfere with $B$, so we can just perform $\mu_{k}$ on $\tilde{D}_{k}$. If $\mu_{k}$ is one of the moves $\mathrm{R}_{\mathrm{I}}^{ \pm 1}, \mathrm{~T}_{\mathrm{I}^{\prime \prime \prime}}, \mathrm{T}_{\mathbb{I I I}^{\prime}}$ or $\mathrm{T}_{\mathrm{V}^{\prime \prime}}$ then again it does not interfere with $B$, and we can perform the appropriate replacement from Figg. 25 or 26, getting a move from $\tilde{D}_{k}$ to $\tilde{D}_{k+1}$. If $\mu_{k}$ is a move of type $\mathrm{T}_{\mathbb{I}^{\prime \prime}}^{ \pm 1}$ or $\mathrm{T}_{\mathrm{I}^{\prime \prime}}^{ \pm 1}$ then it may interfere with $B$. However, since it does not create or destroy cusps, $\mu_{k}$ can be translated on $\tilde{D}_{k}$ as a combination of moves which do not involve cusps, i.e. allowed from the statement. We are only left to deal with the case where $\mu_{k}$ is one of the moves $\mathrm{T}_{\mathrm{I}^{\prime \prime \prime}}^{-1}, \mathrm{~T}_{\mathrm{II}^{\prime}}^{-1}$ or $\mathrm{T}_{\mathrm{V}^{\prime \prime}}^{-1}$ which destroy cusps. By construction the cusp(s) to be destroyed still appear in $\tilde{D}_{k}$ as smoothed cusps within shadowed boxes, so we can destroy them also from $\tilde{D}_{k}$ by means of allowed moves.

The crucial properties 3 and $⿴$ thold by construction, and the conclusion eventually follows.

\section{A calculus for pseudo-Legendrian links in combed manifolds}

We will deal in this section with the set PLeg of equivalence classes of triples $(M, v, L)$ already described in the introduction. Its combinatorial counterpart will be given by the set

$$
\mathcal{L}=\{(P, D): P \in \mathcal{B}, D \in \mathcal{D}(P)\}
$$

where $\mathcal{B}$ is as in Section 2 and $\mathcal{D}(P)$ is as in Section 3. The reconstruction map $(P, D) \mapsto L(P, D)$ is here defined by noting that $D$ defines a link transversal to $v(P)$ in $M(P)$, and hence also a link transversal to $\widehat{v}(P)$ in $\widehat{M}(P)$. According to what we stated after Proposition 2.3 we will actually drop from $\mathcal{B}$ the sliding-MP-rigid spines, and ignore the snake move.

If for a fixed $P$ we consider the effect on $L(P, D)$ of the $\mathrm{C}^{1}$-Turaev moves on $D$, we see that the class of $L(P, D)$ is in general modified by the first Reidemeister move $\mathrm{R}_{\mathrm{I}^{\prime}}$ of Fig. 18 (see also Section 5), but not by the other moves, which we will therefore call pseudo-Legendrian Turaev moves. Other moves which obviously do not change $L(P, D)$ up to equivalence are the sliding-MP-moves on $P$ which do not involve $D$ (these moves permit to follow $D$ along the modification of $P$, so they are well-defined for pairs). It is not hard to see that before performing a sliding-MP-move on $P$ it is always possible to modify $D$ by pseudo-Legendrian Turaev moves to a diagram which 
is not involved in the sliding-MP-move, and the diagram after the sliding-MP-move is well-defined up to pseudo-Legendrian Turaev moves. For this reason we will freely speak of sliding-MP-moves also for pairs. The following will be established below.

Theorem 4.1. The map $L: \mathcal{L} \rightarrow$ PLeg is surjective, and the equivalence relation defined by $L$ is generated by pseudo-Legendrian Turaev moves and sliding-MP-moves.

Fixed-spine statement Recall from the definition that the map $L$ of the statement of Theorem 4.1 involves the passage from $P$ to $(M(P), v(P))$ and then to $(\widehat{M}(P), \widehat{v}(P))$. As already pointed out, this is necessary if one wants to be able to deal with nontraversing fields. However, if one happens to have a concave traversing field, one can directly encode this field by a spine, without first removing a ball, and one can investigate how isotopy of links transversal to the field reflects on link diagrams on the spine. The following is shown below:

Proposition 4.2. Let $P$ be a branched spine. Fix a representative of $(M(P), v(P))$ and an embedding of $P$ in $M(P)$ transversal to $v(P)$. Then every link transversal to $v(P)$ is represented by a $\mathrm{C}^{1}$ diagram on $P$. Moreover two $\mathrm{C}^{1}$ diagrams define the same link up to isotopy through links transversal to $v(P)$ if and only if they are related by pseudo-Legendrian Turaev moves.

From fixed to variable spine We show in this paragraph how to deduce Theorem 4.1 from Proposition 1.2. First of all, to prove surjectivity, we consider a triple $(M, v, L)$ representing an element of PLeg. Using a normal section as in Proposition 2.4, we can obtain a spine $P \in \mathcal{B}$ which encodes the equivalence class of $(M, v)$ in the sense of Theorem 2.1. Moreover $P$ comes with an embedding in $M$ transversal to $v$. Now, a neighbourhood of $P$ can be identified to $M(P)$ and its complement is isomorphic to $B_{\text {triv }}^{3}$. Using the flow generated by $v$ in this ball we can now isotope $L$ through links transversal to $v$ to a link which lies in $M(P)$, and the first assertion of Proposition 4.2 implies that $L$ is represented by a diagram $D$ on $P$. Summing up, we see that $(M, v, L)$ is represented by $(P, D)$, and surjectivity of $L$ is proved.

To conclude we must now show that two pairs $\left(P_{0}, D_{0}\right)$ and $\left(P_{1}, D_{1}\right)$ are equivalent via pseudo-Legendrian Turaev moves when $L\left(P_{0}, D_{0}\right)=L\left(P_{1}, D_{1}\right)$. Spelling out the relation of pseudo-Legendrian isotopy, which defines PLeg, we assume that $P_{0}$ and $P_{1}$ embed in the same manifold $M$ and that there exist a field homotopy $\left(v_{t}\right)_{t \in[0,1]}$ and a link isotopy $\left(L_{t}\right)_{t \in[0,1]}$ on $M$ such that:

1. for $i=0,1$, the link $L_{i}$ is the one defined by $D_{i}$, and the field $v_{i}$ is positively transversal to $P_{i}$ and restricts to $B_{\text {triv }}^{3}$ on the complement of $P_{i}$;

2. $L_{t}$ is transversal to $v_{t}$ for all $t$. 




Figure 27: The annulus $A$.

Given $t \in[0,1]$, we note that for $|t-s| \ll 1$ the link $L_{s}$ is transversal to $v_{t}$, and that a branched spine for $v_{t}$ (in the sense repeatedly used above) is a branched spine also for $v_{s}$. So we can subdivide $[0,1]$ into subintervals $\left[t_{i-1}, t_{i}\right]$ so that:

1. $L_{t_{i-1}}$ is isotopic to $L_{t_{i}}$ through links transversal to $v_{t_{i}}$;

2. $v_{t_{i}}$ has a spine $P_{t_{i}}$ which is also a spine for $v_{t_{i-1}}$.

Now let $D_{t_{i}}$ be a diagram for $L_{t_{i}}$ on $P_{t_{i}}$. Since both $P_{t_{i-1}}$ and $P_{t_{i}}$ are spines for $v_{t_{i}}$, we can transform $P_{t_{i-1}}$ into $P_{t_{i}}$ via sliding-MP-moves. Using pseudo-Legendrian Turaev moves we can now follow $D_{t_{i-1}}$ along this sequence of moves, getting a diagram $D_{t_{i}}^{\prime}$ on $P_{t_{i}}$. Since the sequence of sliding-MP-moves can be realized in $M$ so that each spine of the sequence is a branched spine for $v_{t_{i}}$, we deduce that the link defined by $D_{t_{i}}^{\prime}$ is isotopic to $L_{t_{i-1}}$, and hence to $L_{t_{i}}$, through links transversal to $v_{t_{i}}$. Proposition 4.2 now implies that $D_{t_{i}}^{\prime}$ and $D_{t_{i}}$ are related by pseudo-Legendrian Turaev moves on $P_{t_{i}}$. This shows that $\left(P_{t_{i}}, D_{t_{i}}\right)$ is obtained from $\left(P_{t_{i-1}}, D_{t_{i-1}}\right)$ via the moves of the statement, and the conclusion follows by iteration.

Fixed-spine proof We will establish now Proposition 4.2, writing just $M$ and $v$ for $M(P)$ and $v(P)$. For the sake of simplicity we will assume that $v$ is tangent to $\partial M$ along only one curve (denoted by $\gamma$ ), but our arguments extends almost verbatim to the general case of more than one curve.

We fix in $M$ an annulus $A$ which connects $\gamma$ to $S(P)$ as shown in a cross-section in Fig. 27. Note that $A$ is almost but not quite embedded: it has double point at the vertices of $P$. Since we will only need to consider $A$ locally and away from vertices of $P$, this fact will not disturb us. We choose coordinates $(\rho, \theta) \in[0,1] \times[0,2 \pi]$ on $A$, where $\rho=0$ corresponds to $S(P)$ and $\rho=1$ to $\gamma$. Near $A$ we can also define a coordinate $z \in[-\varepsilon, \varepsilon]$ by integrating $v$.

Now let $L$ be transversal to $v$, and assume by general position that $L$ intersects $A$ only at points with $0<\rho<1$, that no two such intersections have the same coordinate $\theta$, and that at all the intersections the tangent direction to $L$ has non-zero components 


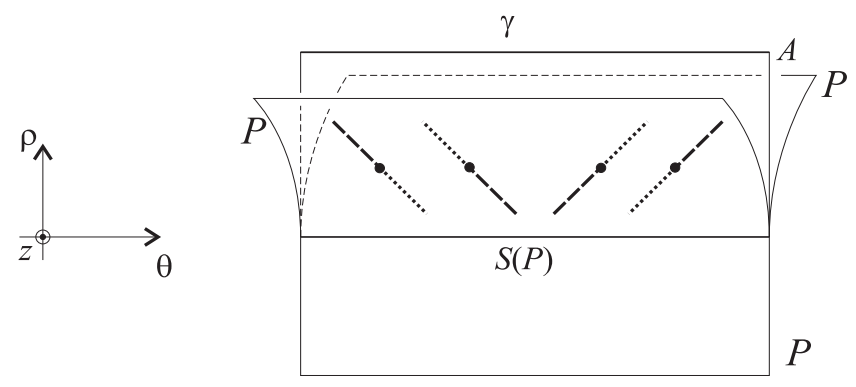

Figure 28: Types of points in $L \cap A$.

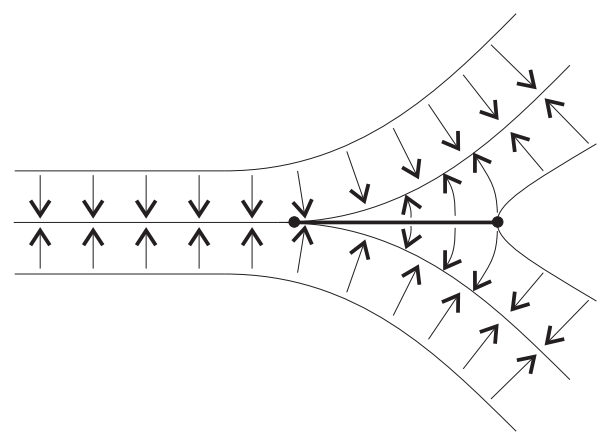

Figure 29: Projection onto $P$.

in all three coordinates $\rho, \theta, z$. Depending on the sign of these components, we can divide the points of $L \cap A$ into four types, shown in Fig. 28 ( $L$ is dashed when it lies over $A$ and dotted when it lies under $A$ ). We consider now the projection $\pi$ of $M \backslash A$ onto $P$ along the orbits of $v$, as shown in Fig. 29. Of course $L \backslash A$ locally projects to a $\mathrm{C}^{1}$-strand on $P$, and by general position we can assume that $\pi(L \backslash A)$ locally appears as a $\mathrm{C}^{1}$-diagram. We are only left to extend the diagram at the points of $L \cap A$, which we do locally in Fig. 30. The top part of this figure actually refers to a simplified situation, because other strands of $L$ already projected on $P$ may locally interfere. We show at the bottom of the same figure in one example how to deal with this fact. The resulting diagram of course represents $L$, and we have proved the first assertion in Proposition 4.2. To prove the second assertion we must now examine an isotopy of $L$ through links transversal to $v$, and hence examine first-order violations of genericity of $L$ with respect to $A$ and $\pi$. All the elementary accidents which do not involve $A$ of course correspond to pseudo-Legendrian Turaev moves. We are left to deal with the following accidents:

1. $L$ intersects $A$ at a point of $S(P) \subset \partial A$;

2. at a point of $L \cap A$, the tangent direction to $L$ has vanishing $\rho$-coordinate;

3. similarly, with the $\theta$-coordinate; 




Figure 30: Completion of the diagram at points of $L \cap A$.

4. similarly, with the $z$-coordinate.

In Fig. 31 we show the situation just before and just after each of these accidents, and we analyze the corresponding transformations of the diagrams constructed as in Fig. 30. In all cases one easily sees that indeed the transformation is generated by pseudo-Legendrian Turaev moves: the number of moves needed is respectively one, zero (isotopy within regions), three and two. By simplicity in Fig. 31 we have ignored the possible interference of other strands of $L$ projected on $P$, but the conclusion is valid anyway (some Reidemeister moves must be added in the general case).

\section{$5 \quad$ Applications and speculations}

In this section we will discuss some consequences of the calculi described above, and mention some natural questions and problems which we put forward for further investigation. The section is split into two subsections.

\subsection{Winding number, torsion, and finite-order invariants}

In this section we employ our realizations of Fram and PLeg in connection with winding number, Maslov index, torsion, and finite-order invariants of pseudo-Legendrian knots.

Relative winding number We spell out in this paragraph the analogue of Trace's result [15] on knot diagrams in $\mathbb{R}^{3}$. We confine ourselves to knots for the sake of simplicity, but essentially the same holds for links.

Proposition 5.1. Let $\left(v_{0}, K_{0}\right)$ and $\left(v_{1}, K_{1}\right)$ be pseudo-Legendrian pairs in a manifold $M$, where $K_{0}$ and $K_{1}$ are oriented knots. Assume that $v_{0}$ and $v_{1}$ are homotopic relatively 

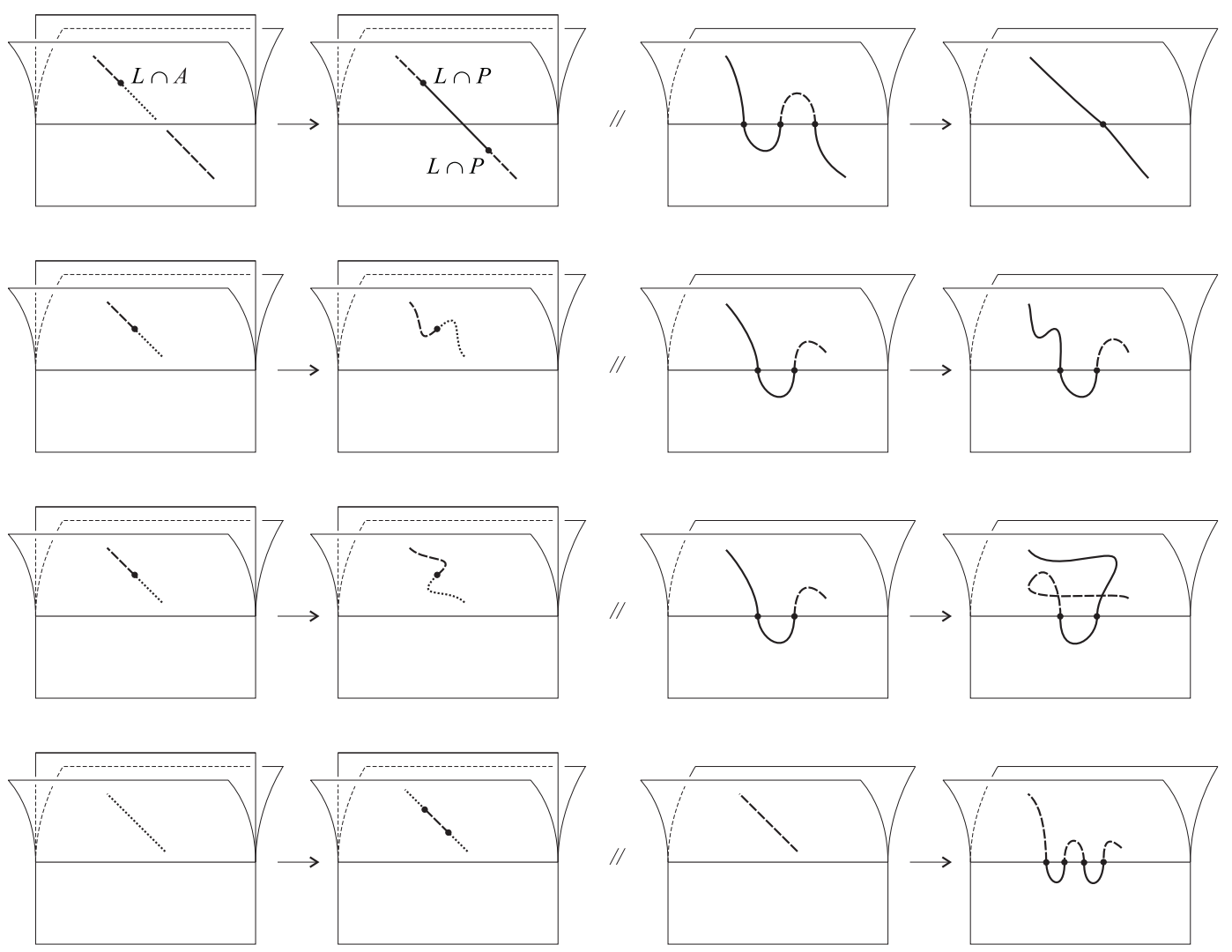

Figure 31: Elementary accidents involving $A$ along an isotopy of $L$. 

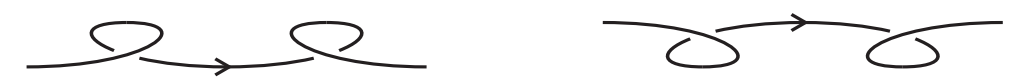

Figure 32: A positive and a negative double curl (here the field points upwards and the orientation is consistent with the orientation of 3-space).



Figure 33: Replacing a non-pseudo-Legendrian move.

to $\partial M$, and that $K_{0}$ and $K_{1}$ are isotopic as oriented framed knots. Then, up to pseudoLegendrian isotopy on $\left(v_{1}, K_{1}\right)$, we can assume that $v_{1}=v_{0}$ and that $K_{1}$ differs from $K_{0}$ only within a region of $\left(M, v_{0}\right)$ isomorphic to $\left(\mathbb{R}^{3}, \partial / \partial z\right)$, where $K_{0}$ is a straight horizontal line and $K_{1}$ has either some positive or some negative double curls (shown in Fig. 3g).

Proof of 5.1. Choose branched spines $P_{0}$ and $P_{1}$ of $v_{0}$ and $v_{1}$ according to Proposition 2.4, and use Proposition 4.2 to represent $K_{0}$ and $K_{1}$ on $P_{0}$ and $P_{1}$ respectively by $\mathrm{C}^{1}$-diagrams $D_{0}$ and $D_{1}$. Since $v_{0}$ and $v_{1}$ are homotopic, a sequence of sliding-MP moves connects $P_{1}$ to $P_{0}$. Following $D_{1}$ along this sequence of moves we get a pseudoLegendrian isotopy, so we can assume that $v_{1}=v_{0}$ and $P_{1}=P_{0}$. Now $D_{0}$ and $D_{1}$ define on $P_{0}$ framed-isotopic oriented knots, so by Theorem 3.1 they are related by $\mathrm{C}^{1}$-Turaev moves. If along the sequence of moves there is no $\mathrm{R}_{\mathrm{I}^{\prime}}$, we deduce pseudoLegendrian equivalence of $K_{0}$ and $K_{1}$. If however there is some $\mathrm{R}_{\mathrm{I}^{\prime}}$, we replace it by a pseudo-Legendrian move as shown in Fig. 33. One easily sees that this replacement can be done consistently along the sequence of moves. The result is a pseudo-Legendrian isotopy between $D_{1}$ and a diagram which differs from $D_{0}$ only for some double curls. These double curls can of course be slid to be consecutive. Now, it is precisely the content of [15] that up to moves $R_{I I}$ and $R_{\text {III }}$ there are only the types of double curls shown in Fig. 32, and that a positive and a negative double curl cancel out. 5.1

Under the assumptions of the previous proposition one may be tempted to define a relative winding number $w\left(K_{1}, K_{0}\right)$ as the (algebraic) number of double curls by which the diagram of $K_{1}$ differs from the diagram of $K_{0}$. This number is however not well-defined in general, as one easily sees in $S^{2} \times S^{1}$ with vector field parallel to the $S^{1}$-factor, because in this case a double curl on a diagram contained in $\mathbb{R}^{2}=S^{2} \backslash\{\infty\}$ can always be removed by isotoping the diagram through $\infty$. This seems to suggest that not $w\left(K_{1}, K_{0}\right)$, but maybe $w\left(K_{1}, K_{0}\right) \cdot\left[\mu_{K_{0}}\right] \in H_{1}\left(E\left(K_{0}\right) ; \mathbb{Z}\right)$ is well-defined, where $E\left(K_{0}\right)$ is the exterior of $K_{0}$ and $\mu_{K_{0}}$ is the meridian. We will show this fact under the additional assumption that $K_{0}$ is 'good' (see [4] and below for explanations). 
Torsion invariants and good knots In [4] we have defined the ReidemeisterTuraev torsion of an Euler structure with simple boundary, and we have applied this notion to define the torsion of pseudo-Legendrian knots. As an absolute invariant torsion contains a sort of lift of the classical Alexander invariant. We will discuss in this paragraph the information carried by torsion as a relative invariant of two pseudoLegendrian framed-isotopic knots $K_{0}, K_{1}$ in the same concave combed manifold $(M, v)$. We recall from [4] that this information is most easily expressed when $K_{0}$ has the property of being good. Goodness depends only on the isotopy class of $K_{0}$ as a framed knot, and it means that a certain quotient of the mapping class group of $E\left(K_{0}\right)$ acts trivially on the space of Euler structures on $E\left(K_{0}\right)$. We omit the precise definition here, but we recall that many knots indeed are good (for instance, all are good if $M$ is a homology sphere, and most hyperbolic knots are good).

When $K_{0}$ is good, the information carried by torsion as a relative invariant depends only on $\alpha\left(\left.v\right|_{E\left(K_{0}\right)}, f\left(\left.v\right|_{E\left(K_{1}\right)}\right) \in H_{1}\left(E\left(K_{0}\right) ; \mathbb{Z}\right)\right.$, where $f \in \operatorname{Diff}_{0}(M)$ maps $K_{1}$ to $K_{0}$ as framed knots, and $\alpha$ is the first obstruction for two vector fields to be homotopic relative to the boundary. So the next result means that for good knots the relative winding number gives a well-defined invariant, and all the information torsion can capture is contained in the relative winding number. The statement involves all the assumptions and notations of the present and previous paragraph.

Proposition 5.2. $\alpha\left(\left.v\right|_{E\left(K_{0}\right)}, f\left(\left.v\right|_{E\left(K_{1}\right)}\right)=w\left(K_{1}, K_{0}\right) \cdot\left[\mu_{K_{0}}\right] \in H_{1}\left(E\left(K_{0}\right) ; \mathbb{Z}\right)\right.$.

Proof of 5.2. We note first that $w\left(K_{1}, K_{0}\right)$ and $\left[\mu_{K_{0}}\right]$ depend on the choice of an orientation on $K_{0}$, but their product does not, so the statement makes sense. For the proof, note that by goodness we can just assume that $K_{0}$ and $K_{1}$ differ as in the statement of Proposition 5.1, and that $f$ is supported on a neighbourhood of the region where $K_{0}$ and $K_{1}$ differ. The conclusion then follows directly from Proposition 2.17 of 迎.

Even if we have not discussed finite-order invariants yet, we note here that Proposition 5.2 implies that torsion is a weaker invariant than the finite-order ones for Legendrian knots in a given homotopy class of Legendrian immersions. To our knowledge the only known examples of framed-isotopic knots distinguished by such invariants are those due to Tchernov [14], and we believe that they are all good (at least, they certainly are good when the ambient manifold is $S^{2} \times S^{1}$ ). Now one sees that in all of Tchernov examples $w\left(K_{1}, K_{0}\right) \cdot\left[\mu_{K_{0}}\right]=0$, so torsion definitely cannot distinguish. On the other hand, the definition of torsion does not require fixing a homotopy class of Legendrian immersions, so torsion and finite-order invariants are in some sense complementary.

We will state in the rest of this paragraph some interesting consequences of Proposition 5.2, always assuming the knots involved to be good. For simplicity, as in Proposition 5.2, we stick to knots transverse to a given field $v$ on a given $M$, but we remind that the relation of pseudo-Legendrian isotopy also involves a homotopy of $v$. 
Corollary 5.3. Under the same assumptions as in Proposition 5.9, suppose furthermore that $\left[\mu_{K_{0}}\right]$ has infinite order in $H_{1}\left(E\left(K_{0}\right) ; \mathbb{Z}\right)$, so $w\left(K_{1}, K_{0}\right) \in \mathbb{Z}$ is well-defined. Then the following facts are pairwise equivalent:

1. $w\left(K_{1}, K_{0}\right)=0$;

2. $K_{0}$ and $K_{1}$ have trivial relative torsion invariants;

3. $K_{0}$ and $K_{1}$ are pseudo-Legendrian isotopic.

Proof of 5.3. Equivalence of (1) and (3) follows from the definition of $w\left(K_{1}, K_{0}\right)$. Implication (17) $\Rightarrow(2)$ follows from Proposition 5.2, and the opposite implication follows by taking the torsion associated to a representation $\varphi$ of $H_{1}\left(E\left(K_{0}\right) ; \mathbb{Z}\right)$ such that $\varphi\left(\left[\mu_{K_{0}}\right]\right)$ has infinite order (see [4] for details). 5.3

If $M$ is a homology sphere and $K$ is a pseudo-Legendrian knot in $(M, v)$ we have shown in [4] that the rotation number $\operatorname{rot}_{v}(K)$, also called Maslov index, can be defined just as in the case where $K$ is Legendrian in a contact structure. Now:

Lemma 5.4. If $M$ is a homology sphere, $K_{0}$ and $K_{1}$ are pseudo-Legendrian in $(M, v)$ and framed isotopic, then $w\left(K_{1}, K_{0}\right)=\frac{1}{2}\left(\operatorname{rot}_{v}\left(K_{1}\right)-\operatorname{rot}_{v}\left(K_{0}\right)\right)$.

(Concerning the statement, note that $\operatorname{rot}_{v}\left(K_{1}\right)-\operatorname{rot}_{v}\left(K_{0}\right)$ must be even if $K_{0}$ and $K_{1}$ are framed-isotopic, otherwise one of $\left\{K_{0}, K_{1}\right\}$ would lift to a closed path in a spin structure on $M$, and the other one would not: a contradiction. A proof is easily obtained by isotoping $K_{1}$ as stated in Proposition 5.1.)

Lemma 5.4 gives another proof of the fact that $w\left(K_{1}, K_{0}\right) \in \mathbb{Z}$ can be defined when $M$ is a homology sphere. Moreover, it could be used to show goodness of knots in a homology sphere by a more direct argument than that given in 四. We conclude this paragraph by showing the result stated in the introduction and asking a question which naturally arises from it.

Proof of 0.1. Equivalence of (11) and (2) comes from Corollary 5.3. Equivalence of (2) and (3) comes from Lemma 5.4. Equivalence of (3) and (41) follows from Corollary5.3 and the fact that the first homology group of the complement of a knot in $M$ is infinite cyclic and generated by a meridian. Equivalence of (3) and (5) is an application of Gromov's $h$-principle (see [14]).

Question 5.5. Let $(M, v)$ be an arbitrary combed manifold, let $K_{0}$ and $K_{1}$ be pseudoLegendrian in $(M, v)$ and framed-isotopic, and assume that they are homotopic through pseudo-Legendrian immersions. Does this imply that $w\left(K_{1}, K_{0}\right) \cdot\left[\mu_{K_{0}}\right]=0$ ? (We do not think that the opposite implication can be true in general, in particular when $\left[\mu_{K_{0}}\right]$ has finite order.) 
Absolute winding number We concentrate in this paragraph on fields $v$ such that $\mathcal{E}\left(v^{\perp}\right)=0$, where $\mathcal{E}$ denotes the Euler class and the choice of the metric is of course immaterial. Condition $\mathcal{E}\left(v^{\perp}\right)=0$ is equivalent to the existence of another non-vanishing field $x$ always transversal to $v$. Since the ambient manifold is oriented, this is also equivalent to the fact that $v$ extends to a framing $(v, x, y)$, i.e. a global trivialization of the tangent bundle to $M$. Assume now that $K$ is an oriented knot transversal to $v$. Then, taking the projection of the tangent vector to $K$ on the unit sphere of the $(x, y)$-plane, and computing the degree, we can define a rotation number $\operatorname{rot}_{(v, x)}(K)$.

Remark 5.6. $\operatorname{rot}_{(v, x)}(K)$ is invariant under simultaneous homotopy $\left(v_{t}, x_{t}\right)$ and isotopy $\left(K_{t}\right)$ such that $x_{t}$ and $v_{t}$ are transversal to $v_{t}$ for all $t$. Moreover $\operatorname{rot}_{(v, x)}(K)$ is independent of $x$ when $M$ is a homology sphere, and it equals the Maslov index already discussed above.

Assume now that $K_{0}$ and $K_{1}$ are both transversal to $v$. Within the proof of Proposition 5.1 we have shown that $K_{1}$ can be isotoped through knots transversal to $v$ to a knots which differs from $K_{0}$ by double curls only.

Remark 5.7. $\operatorname{rot}_{(v, x)}\left(K_{1}\right)-\operatorname{rot}_{(v, x)}\left(K_{0}\right)$ is independent of $x$ and equals twice the number of double curls by which $K_{0}$ and $K_{1}$ differ, up to isotopy transversal to $v$.

The previous remark shows that the relative winding number is well-defined (without any assumption on the knots) if one restricts to knots transversal to a given $v$ with $\mathcal{E}\left(v^{\perp}\right)=0$, and one views the knots up to isotopy transversal to $v$ (as opposed to pseudo-Legendrian isotopy, which involves also a homotopy of $v$ ). More on the difference between transversal isotopy and pesudo-Legendrian isotopy will be said below.

Remark 5.8. Combining the previous two remarks one gets yet another proof that the relative winding number is well-defined in $\mathbb{Z}$ up to pseudo-Legendrian isotopy in a homology sphere.

Finite-order invariants We formally state and motivate in this paragraph the conjecture announced in the introduction. Let $\xi$ be an oriented contact structure on $M$ (which we assume to be closed by simplicity), and let $v$ be a field positively transversal to $\xi$. Consider the spaces $\operatorname{Leg}(M, \xi), \operatorname{PLeg}^{\text {weak }}(M, v)$ and $\operatorname{Fram}(M)$ of $\xi$-Legendrian, $v$ transverse, and framed knots in $M$, with the appropriate equivalence relations (namely $\xi$-Legendrian, pseudo-Legendrian, and framed isotopy). Enlarge these spaces by allowing immersions of $S^{1}$ rather than embeddings, and take path-connected components $\mathfrak{l}, \mathfrak{p}$ and $\mathfrak{f}$, with $\mathfrak{l} \subset \mathfrak{p} \subset \mathfrak{f}$. (Concerning PLeg, note that a path is a family $\left(K_{t}, v_{t}\right)_{t \in[0,1]}$ with $v_{0}=v_{1}=v$.) Given an Abelian group $A$ one can define, using the customary VassilievGoussarov skein relations, the spaces $V_{\mathfrak{l}}^{n}(A), V_{\mathfrak{p}}^{n}(A)$ and $V_{\mathfrak{f}}^{n}(A)$ of $A$-valued order- $n$ invariants under Legendrian, pseudo-Legendrian and framed isotopy respectively. Since 
a Legendrian isotopy is pseudo-Legendrian, and a pseudo-Legendrian isotopy is framed, using restrictions we get a commutative diagram of homomorphisms:

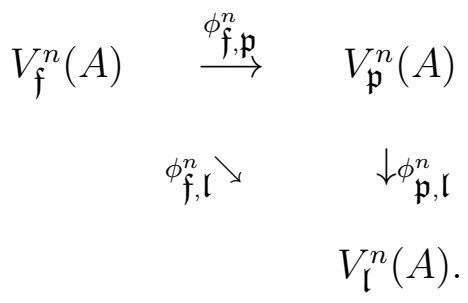

Tchernov's arguments [14] imply that all three $\phi$ 's are always injective, and our conjecture is that $\phi_{\mathfrak{p}, \mathfrak{l}}^{n}$ is always an isomorphism. By [14] again, the conjecture is equivalent to showing that every finite-order Legendrian invariant is automatically invariant also under pseudo-Legendrian isotopy. The generalized Fuchs-Tabachnikov theorem (see [8] and [14]) states that $\phi_{\mathfrak{f}, \mathfrak{l}}^{n}$ is an isomorphism in many cases (e.g. if $M$ is a homology sphere), so $\phi_{\mathfrak{p}, \mathfrak{l}}^{n}$ is also an isomorphism in these cases. Tchernov has provided the only known examples in which $\phi_{\mathfrak{f}, \mathfrak{l}}^{n}$ is not an isomorphisms, namely he has exhibited elements of $V_{\mathfrak{l}}^{n}(A)$ which do not lift to $V_{\mathfrak{f}}^{n}(A)$. Our impression is that these elements do lift to $V_{\mathfrak{p}}^{n}(A)$, which would imply that $\phi_{\mathfrak{p}, \mathfrak{l}}^{n}$ is indeed an isomorphism in all known cases. Truthness of our conjecture would imply that Legendrian finite-order invariants are only sensitive to the homotopy class of a contact structure, and in particular that they cannot capture tightness.

\subsection{Pseudo-Legendrian vs. Legendrian knots}

After the work of Eliashberg [7], we know that on a closed manifold an overtwisted contact structure is determined up to isotopy by its homotopy class as a plane field. We discuss in this section the extent to which this fact extends in presence of a pseudoLegendrian link. We start with an open question which arises from the results of Section 4 and will lead us to the connection with overtwisted contact structures.

Fixed vs. variable spine for pseudo-Legendrian links We will adopt in this paragraph the viewpoint which allows to dismiss automorphisms of manifolds, fixing $M$ and considering spines and moves embedded in $M$, as explained after the statement of Proposition 2.3.

Theorems 2.1 and 4.1 and Proposition 4.2 leave the following question open: given an embedded spine $P \subset M$ representing a concave combing on $M$, what intrinsic topological object is represented by $\mathrm{C}^{1}$-diagrams on $P$ up to pseudo-Legendrian Turaev moves? Let us introduce some notation to formalize the situation. We denote by $\mathcal{D}^{\text {PLeg }}(P)$ the set of equivalence classes of $\mathrm{C}^{1}$-diagrams on $P$ up to pseudo-Legendrian Turaev moves. We also fix a representative $v$ of the combing carried by $P$ (so, $v$ is positively transversal to $P$ and restricts to $B_{\text {triv }}^{3}$ on the complement of $P$ ). We consider 
now the set of links in $M$ transversal to $v$, and we denote by $\operatorname{PLeg}^{\text {weak }}(M, v)$ the quotient space under the relation of existence of a pseudo-Legendrian isotopy, i.e. a path $\left(L_{t}, v_{t}\right)_{t \in[0,1]}$ as usual, with $v_{0}=v_{1}=v$. We also denote by $\operatorname{PLeg}^{\text {strong }}(M, v)$ the (bigger) quotient obtained by forcing $\left(v_{t}\right)$ to be constant. So $\operatorname{PLeg}^{\text {strong }}(M, v)$ is just the set of equivalence classes of links transversal to $v$. Using Proposition 4.2 one sees that the operation of turning a diagram into a link defines a bijection

$$
\psi^{\text {strong }}: \mathcal{D}^{\text {PLeg }}(P) \rightarrow \mathrm{PLeg}^{\text {strong }}(M, v) .
$$

(This is not quite the content of Proposition 4.2, because here $(M, v)$ is $(\widehat{M}(P), \widehat{v}(P))$ rather that $(M(P), v(P))$, but a link isotopy can be modified to avoid a $B_{\text {triv }}^{3}$, and the conclusion follows.)

Bijectivity of $\psi^{\text {strong }}$ is significant if one imagines to have started with the pair $(M, v)$, and to have constructed $P$ from a normal section of $v$, as in Proposition 2.4. It is however less significant if one assumes only $P$ to be given from the beginning, because in this case $v$ is actually well-defined only up to homotopy, and fixing a representative looks artificial. The natural map to consider is in this case

$$
\psi^{\text {weak }}: \mathcal{D}^{\text {PLeg }}(P) \rightarrow \mathrm{PLeg}^{\text {weak }}(M, v)
$$

obtained by composition with the projection $\mathrm{PLeg}^{\text {strong }}(M, v) \rightarrow \mathrm{PLeg}^{\text {weak }}(M, v)$. This map is of course surjective, and one can ask whether it is injective or not. Some remarks are in order:

1. Theorem 4.1 implies that if $\psi^{\text {weak }}(D)=\psi^{\text {weak }}\left(D^{\prime}\right)$ then there exists a circular sequence $P=P_{0} \rightarrow P_{1} \rightarrow \cdots \rightarrow P_{n}=P$ of sliding-MP-moves and diagrams $D_{i}, D_{i}^{\prime} \in \mathcal{D}\left(P_{i}\right)$ with $D_{0}=D, D_{n}^{\prime}=D^{\prime}, D_{i} \rightarrow D_{i}^{\prime}$ a pseudo-Legendrian Turaev move, and $D_{i+1}$ the companion of $D_{i}^{\prime}$ through $P_{i} \rightarrow P_{i+1}$. Checking the injectivity of $\psi^{\text {weak }}$ corresponds to the (purely combinatorial) question whether such a sequence $\left(P_{i}, D_{i}\right)$ can be replaced by one with constant $P_{i}$.

2. Using Theorem 2.1 and the fact that $\mathrm{C}^{1}$ diagrams can be followed through slidingMP-moves, one sees quite easily that injectivity of $\psi^{\text {weak }}$ actually depends only on the combing carried by $P$, not on $P$ itself.

3. Injectivity of $\psi^{\text {weak }}$ is equivalent to injectivity of the projection $\operatorname{PLeg}^{\text {strong }}(M, v) \rightarrow$ $\operatorname{PLeg}^{\text {weak }}(M, v)$, a purely topological question. Injectivity of this projection may appear very unlikely at first sight, since it basically corresponds to the fact that a homotopy can be replaced by an isotopy. However one can remark that injectivity of projection depends only on the homotopy class of $v$, rather than $v$ itself, so one can assume that $v$ is transversal to an overtwisted contact structure. For overtwisted structures, after the work of Eliashberg [7], it is indeed true that homotopy implies isotopy, but the presence of the link of course somewhat modifies the situation. We will expound this theme in the next paragraph. 
Overtwisted structures and overtwisted knot complements We fix in this paragraph an overtwisted contact structure $\xi$ on $M$ (which we assume to be closed by simplicity) and a field $v$ positively transversal to $\xi$. We will denote by $\operatorname{Leg}(M, \xi)$ the space of Legendrian links in $(M, \xi)$ up to Legendrian isotopy. In [⿴囗⿱一一⿻儿口 , having also in mind the facts mentioned in the previous paragraph, we put forward the question of whether the natural map

$$
\operatorname{Leg}(M, \xi) \rightarrow \mathrm{PLeg}^{\text {weak }}(M, v)
$$

is a bijection. A fact implying that this map is not injective in some cases was recently communicated to us by E. Giroux [9]. He was able to construct triples $(M, \xi, K)$ where $\xi$ is overtwisted, $K$ is $\xi$-Legendrian, and $\left.\xi\right|_{M \backslash K}$ is tight. Let us apply a Lutz twist away from $K$ to get a new structure $\xi^{\prime}$ such that $\xi^{\prime}$ is homotopic to $\xi$ as a plane field on $M$, and $\left.\xi^{\prime}\right|_{M \backslash K}$ is overtwisted. Using Eliashberg's classification [7] we consider $\varphi \in \operatorname{Diff}_{0}(M)$ such that $\xi^{\prime}=\varphi^{*}(\xi)$, and define $K^{\prime}=\varphi(K)$. By construction $K$ and $K^{\prime}$ have the same image in $\operatorname{PLeg}^{\text {weak }}(M, v)$, but of course they are inequivalent in $\operatorname{Leg}(M, \xi)$.

To avoid the phenomenon discovered by Giroux we consider in $\operatorname{Leg}(M, \xi)$ the subset $\operatorname{Leg}^{\mathrm{OT}}(M, \xi)$ given by links whose complement is overtwisted. We start by showing:

Proposition 5.9. The natural map $\mathrm{Leg}^{\mathrm{OT}}(M, \xi) \rightarrow \mathrm{PLeg}^{\text {weak }}(M, v)$ is surjective.

Proof of 5.9. Let $L$ be transversal to $v$, and fix a metric on $M$. Let $\eta$ be a positive contact structure near $L$ with $\eta=v^{\perp}$ on $K$ (such an $\eta$ is unique up to isomorphism). Extend $\eta$ to any plane field homotopic to $v^{\perp}$ (and hence to $\xi$ ) on $M$. So $\eta$ is a plane distribution which has a contact zone, and $L$ lies in this contact zone. The technique of Eliashberg [7] now allows to homotope $\eta$ away from its contact zone to an overtwisted contact structure $\xi^{\prime}$. The resulting $\xi^{\prime}$ is now isotopic to $\xi$, again by Eliashberg's result. If $\varphi \in \operatorname{Diff}_{0}(M)$ and $\xi^{\prime}=\varphi^{*}(\xi)$ we define $L^{\prime}=\varphi(L)$. By construction $\left(L^{\prime}, v\right)$ is pseudo-Legendrian isotopic to $(L, v)$, and surjectivity is proved. 5.9

We cannot presently state whether the map $\operatorname{Leg}^{\mathrm{OT}}(M, \xi) \rightarrow \mathrm{PLeg}^{\text {weak }}(M, v)$ is injective or not in general. We only give a partial argument (based on the techniques of Eliashberg again), and mention where the difficulty arises. Assume that $L_{0}$ and $L_{1}$ are $\xi$-Legendrian with overtwisted complements and define equivalent pseudo-Legendrian links. Then there exists a continuous family $\left(L_{t}, \xi_{t}\right)_{t \in[0,1]}$, where $\xi_{0}=\xi_{1}=\xi$ but $\xi_{t}$ is only a plane field for $t \neq 0,1$. Eliashberg's contactization methods for homotopies, together with the uniqueness of contact structures in the neighbourhood of Legendrian links, should in our opinion allow to replace such a $\left(\xi_{t}\right)_{t \in[0,1]}$ by another one in which each $\xi_{t}$ is a contact structure (and still contains $L_{t}$ as a Legendrian link). Applying Gray's theorem we get an isotopy $\left(\varphi_{t}\right)_{t \in[0,1]}$ such that $\xi_{t}=\varphi_{t}^{*}\left(\xi_{0}\right)$. Setting $\tilde{L}_{t}=\varphi_{t}\left(L_{t}\right)$ we get a Legendrian isotopy between $L_{0}$ and $\varphi_{1}\left(L_{1}\right)$. The question whether $\varphi_{1}\left(L_{1}\right)$ is automatically Legendrian isotopic to $L_{1}$, at least for some classes of manifolds, now depends on the analysis of the group $\operatorname{Aut}(M, \xi) \cap \operatorname{Diff}_{0}(M)$, which we leave unsettled for the time being. 


\section{References}

[1] R. Benedetti, C. Petronio, A finite graphic calculus for 3-manifolds, Manuscripta Math. 88 (1995), 291-310.

[2] R. Benedetti, C. Petronio, "Branched Standard Spines of 3-Manifolds", Lecture Notes in Math. n. 1653, Springer-Verlag, Berlin-Heidelberg-New York, 1997.

[3] R. Benedetti, C. Petronio, Branched spines and contact structures on 3-manifolds, Ann. Mat. Pura Appl. (to appear).

[4] R. Benedetti, C. Petronio, Reidemeister torsion of 3-dimensional Euler structures with simple boundary and Legendrian links, preprint math.GT/9907184, submitted.

[5] S. Benvenuti, Hopf algebras and invariants of combed and framed 3manifolds, In: "Knots in Hellas '98," to appear on a special issue of J. Knot Theory Ramif.

[6] B. G. CASLER, An imbedding theorem for connected 3-manifolds with boundary, Proc. Amer. Math. Soc. 16 (1965), 559-566.

[7] Ya. Eliashberg, Classification of overtwisted contact structures, Invent. Math. 98 (1989), 623-637.

[8] D. Fuchs, S. TABaChnikov, Invariants of Legendrian and transverse knots in the standard contact space, Topology 36 (1997), 1025-1053.

[9] E. Giroux, private communication, December 1999.

[10] I. IsHII, Moves for flow-spines and topological invariants of 3-manifolds, Tokyo J. Math. 15 (1992), 297-312.

[11] S. V. Matveev, Transformations of special spines and the Zeeman conjecture, Math. USSR-Izv. 31 (1988), 423-434.

[12] G. Meng, C. Taubes, $\underline{S W}=$ Milnor torsion, Math. Res. Lett. 3 (1996), $661-674$

[13] R. Piergallini, Standard moves for standard polyhedra and spines, Rendiconti Circ. Mat. Palermo 37, suppl. 18 (1988), 391-414.

[14] V. TChennov, Finite order invariants of Legendrian, transverse, and framed knots in contact 3-manifolds, preprint 1999. 
[15] B. Trace, On the Reidemeister moves of a classical knot, Proc. Amer. Math. Soc. 89 (1983), 722-724.

[16] V. G. Turaev, "Quantum Invariants of Knots and 3-Manifolds", de Gruyter stud. in Math. 18, Berlin-New York, 1994.

[17] V. G. Turaev, Euler structures, nonsingular vector fields, and torsion of Reidemeister type, Math. USSR-Izv. 34 (1990), 627-662.

[18] V. G. Turaev, Torsion invariants of Spinc ${ }^{c}$-structures on 3-manifolds, Math. Res. Lett. 4 (1997), 679-695.

[19] V. G. Turaev, A combinatorial formulation for Seiberg-Witten invariants of 3-manifolds, Math. Res. Lett. 5 (1998), 583-598.

benedett@dm.unipi.it

petronio@dm.unipi.it

Dipartimento di Matematica

Via F. Buonarroti, 2

I-56127, PISA (Italy) 